\title{
Effects of Planform Geometry and Pivot Axis Location on the Aerodynamics of Pitching Low Aspect Ratio Wings
}

\author{
Huai-Te $\mathrm{Yu}^{1}$ and Luis P. Bernal ${ }^{2}$ \\ University of Michigan, Ann Arbor,MI,48109 \\ and \\ Michael V. $\mathrm{Ol}^{3}$ \\ U.S. Air Force Research Laboratory
}

\begin{abstract}
We consider aerodynamic forces and flow development for several wing planforms with the same aspect ratio $(A R=4)$ and mean chord, undergoing a constant-rate pitch change between 0 and $45^{\circ}$. The wing planforms studied are rectangular, trapezoidal and triangular, which correspond to taper ratios $1,0.5$ and 0 , respectively. Three pivot axes are considered: leading edge, mid chord and trailing edge. The reduced pitch rate based on chord and free stream speed is $K=0.39$ and the Reynolds number is $R e=8.9 \mathrm{k}$. We consider also the case $K$ $=\infty$ with the same rotation rate as the other cases and zero free stream velocity. Noncirculatory effects are found for leading edge and trailing edge pivot axes, and absent for mid-chord pivot for all wing planforms. The lift and drag coefficients during the constant pitch rate part of the motion increase as the taper ratio decreases due to rotation rate effects, which is contrary to steady flow behavior in which lift and drag coefficients are only functions of aspect ratio, independent of wing planform geometry. Lifting-line theory including rotation rate effects gives reasonable estimation of lift. Flow visualization show primarily 2D flow during the pitch-up motion. The three-dimensional swirl flow in the wake due to tip vortices is observed after the end of the pitch motion. It develops faster for lower tapper ratio wings.
\end{abstract}

\footnotetext{
${ }^{1}$ Ph.D. Student, Department of Aerospace Engineering, University of Michigan, htyu@umich.edu, and AIAA Student Member.

${ }^{2}$ Associate Professor, Department of Aerospace Engineering, University of Michigan, lpb@umich.edu, and AIAA Member.

${ }^{3}$ Aerospace Engineer, Air Vehicles Directorate, AFRL/VAAA, Bldg. 45, 2130 8th St., Wright-Patterson AFB, OH 45433-7542, Michael.Ol@wpafb.af.mil, AIAA Associate Fellow
} 


\section{Nomenclature}

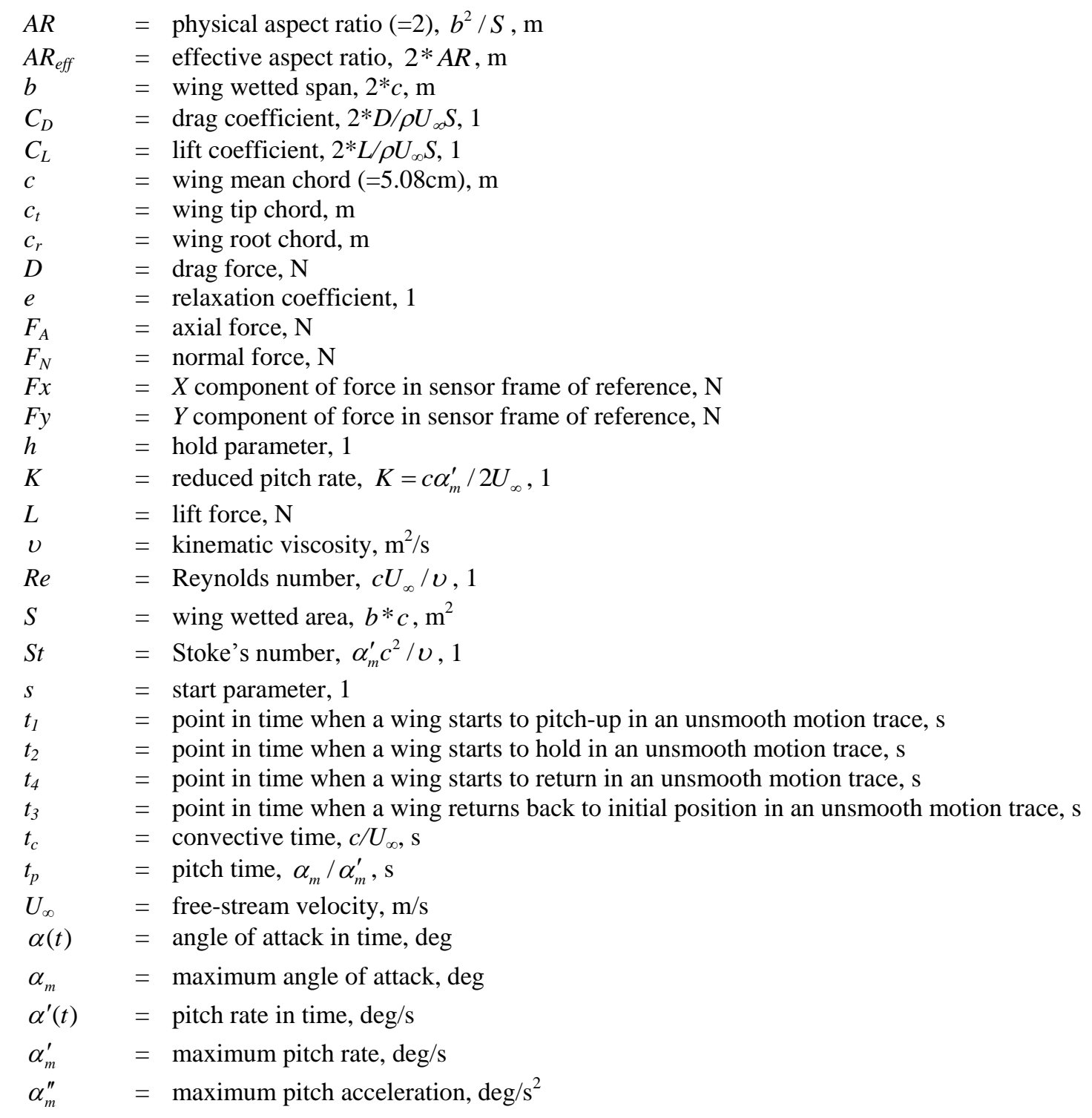

\section{Introduction}

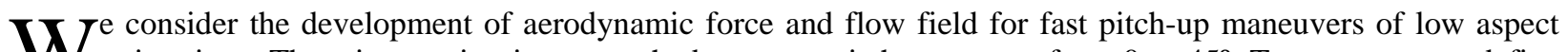
ratio wings. The wing motion is a smoothed constant pitch-rate ramp from 0 to $45^{\circ}$. Two parameters define the flow and wing kinematics: the Reynolds number based on flow speed, mean wing chord and kinematic viscosity; and the Stokes number based on the pitch rate, mean wing chord and kinematic viscosity. This problem has received considerable attention in the past as summarized in Fig. 1. The abscissa of Fig. 1 is the Reynolds number, and the ordinate of Fig. 1 is the Stokes number. As documented in the literature (see references noted in Fig. 1, [1] - [14]) boundary layer separation at the leading edge results in formation of a leading edge vortex (LEV) which remains attached on the suction side of the airfoil and produces high lift. At later times the LEV detaches, and the lift coefficient decreases reaching the steady state value after a several convective times. Also shown in Fig 1 are lines for constant reduced pitch rate, $K=c \alpha_{m}^{\prime} / 2 U_{\infty}$, which denotes the ratio of convective time ( $c / U$ ) to pitch time $\left(\alpha_{m} / \alpha_{m}^{\prime}\right)$, for a given maximum angle of attack $\alpha_{m}=45^{\circ}$. The reduced pitch rate values shown in Fig. 1 represent conditions of interest. For $K=0.393(\pi / 8)$ the duration of the $0-45^{\circ}$ pitching motion equals one convective time, 
which is not sufficient for the LEV to detach. For $K=0.065$ the duration of the pitching motion is 6 convective times which allows the LEV to detach. For $k=2.356$ the pitch up motion is completed in a fraction of the convective time. Clearly these different evolution states of the LEV for different reduced pitch rates must be accompanied by significant changes in the evolution of the wing tip (TV) vortex of finite aspect ratio wings, which may also be affected by pivot axis location and wing planform geometry.

In the present research we explore the aerodynamic force and flow development of finite aspect ratio wings for different planform geometries and pivot axes at reduced pitch rate $K=0.393$. Experiments are reported for fixed aspect ratio flat-plate wings $(A R=4)$ with rounded edges and pivot axis at the leading edge, trailing edge and mid chord. This work extends recent work by the authors and collaborators: Ol et al [14] used flow visualization (dye injection, $\alpha_{m}=20^{\circ}$ and $40^{\circ}$ ) on an infinite wing and a wing with aspect ratio of 2 with rounded edges, Baik et al. [17] reports PIV data for a 2D wing and $\alpha_{m}=90^{\circ}$, Yu et al [18] present direct force measurements and PIV for a 2D wing at $\alpha_{m}=33^{\circ}, 45^{\circ}, 57^{\circ}$; and more recently experiments for a finite wing with $A R=4$ were conducted by $\mathrm{Yu}$ and Bernal [19]. A feature of interest is the formation of an arch vortex reported by Visbal [20] in heaving motion and Yilmaz and Rockwell [21] in pitching motion of flat plate wings at relatively low reduced pitch rate.

\begin{tabular}{|c|c|c|}
\hline$\diamond$ Daley\&Jumper(1984) & $\square$ Helin\&Walker(1985) & $\triangle$ Walker\&Chou(1985) \\
\hline$\times$ Walker et al (1985) & * Strickland\&Graham(1986) & oGraham\&Strickland(1986) \\
\hline + Strickland\&Graham(1987) & +Walker et al (1987) & OJumper et al (1987) \\
\hline * Albertson et al (1988) & $\square$ Jumper et al (1989) & $\Delta$ Shih et al (1992) \\
\hline 口Ol (2009) & $\Delta$ Grandlund et al (2010) & - Baik et al (2010) \\
\hline - Yu et al (2012) & oYilmza\&Rockwell (2012) & - Yu\&Bernal (2013) \\
\hline
\end{tabular}

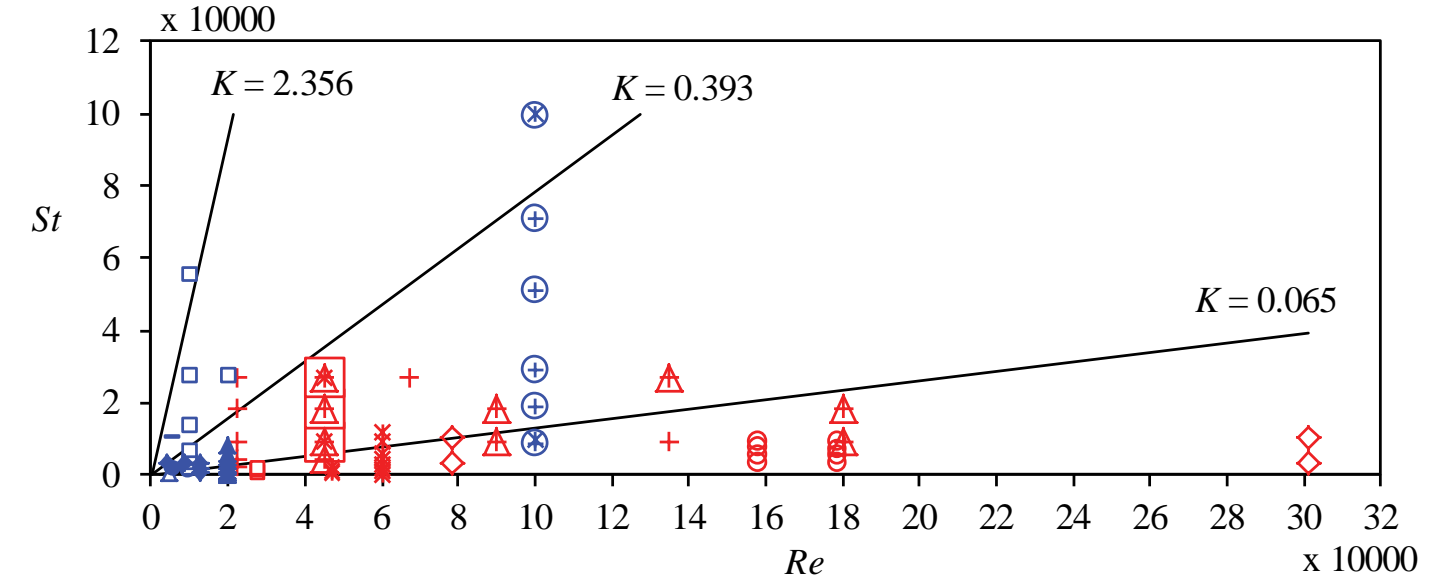

Figure 1. Summary of test cases reported in the literature in terms Reynolds number and Stokes number Experimental Apparatus. Red symbols represent experiments in wind tunnel, and blue symbols represent experiments in water tunnel.

\section{A. Wing Configuration and Water Tunnel}

The experiments were conducted in the low-turbulence water tunnel at University of Michigan with cross section $61 \mathrm{~cm}$ wide and $61 \mathrm{~cm}$ height. This tunnel produces steady free stream velocity from $5 \mathrm{~cm} / \mathrm{s}$ to $40 \mathrm{~cm} / \mathrm{s}$.

Finite aspect ratio wings with effective aspect ratio 4 were employed. Planform geometries include: a rectangular wing, two trapezoidal wings (isosceles and right), and two triangular wings (isosceles and right), as shown in Fig. 2. The three holes near the top edge are used to attach the wings to a sensor adapter, which in turn is attached to the force transducer; these mounting holes are aligned with the pitch axis which also coincident with the axis of the force transducer. The sensor measures forces in the chord direction (x-axis), normal to the wing surface (y-axis) and in the span direction (z-axis) and corresponding torques. Three pivot axes are implemented, including leading edge (LE) pivot, mid-chord (ME) pivot, and trailing edge (TE) pivot. The right trapezoidal and triangular wings are used for the LE and TE pivot axes tests. The edge normal to the wing root chord (no sweep) is the pivot axis for all these cases and the wing is rotated $180^{\circ}$ to change from the LE to the TE pivot axis. The force transducer coordinate 
system is the same for the LE and ME pivot axis, and is rotated $180^{\circ}$ for the TE pivot axis. The coordinates of force transducer are the same for LE and ME using difference pitch-axis alignment. A picture of force measurement setup using the trapezoidal wing for LE pivot is shown in Fig. 3. The wing model is partially submerged in the water channel with the free surface providing a plane of symmetry for the flow.

The force sensor is a Nano 43 Force/Torque sensor manufactured by ATI Industrial Automation. The sensor's maximum calibrated load is $18 \mathrm{~N}$ and the resolution is $1 / 256 \mathrm{~N}$, in all three axes. The force sensor is attached to a rotary table Velmex (B4818TS) driven by a stepper motor, which has a resolution of $20 \mathrm{deg} / \mathrm{s}$ and maximum pitch rate of $200 \mathrm{deg} / \mathrm{s}$. Both the force sensor and the rotary table are located above the water surface. All wings are flat plates with mean-chord length of 2" and 2 mean-chords immersed in water. The thickness is 0.125 " (i.e. $6.25 \%$ of chord) and all edges are rounded. They are made of plexiglass sheet, mounted vertically at the center of the water channel. The wings are attached to the tool side of the sensor with an aluminum adapter designed to minimize the mass of the system. The mass attached to the sensor for all the wings are tabulated in Table 1, the total mass contributed to sensor is not more than 46.2 grams, which includes the wing itself, a sensor adapter and screws. Because of the small mass, inertia forces and static weight of the model are very small which makes static calibration unnecessary. The forces measured by the sensor $(F x$ and $F y$ ) are first converted to axial and normal forces, $F_{A}$ and $F_{N}$, and then to components in the laboratory frame of reference using Eq. (1) - (4) for lift force $(L)$ and drag force $(D)$ and their corresponding force coefficients.

$$
\begin{gathered}
L=-F_{A} \sin \alpha+F_{N} \cos \alpha \\
D=F_{A} \cos \alpha+F_{N} \sin \alpha \\
C_{L}=L /\left(\rho U_{\infty}{ }^{2} S / 2\right) \\
C_{D}=D /\left(\rho U_{\infty}{ }^{2} S / 2\right)
\end{gathered}
$$
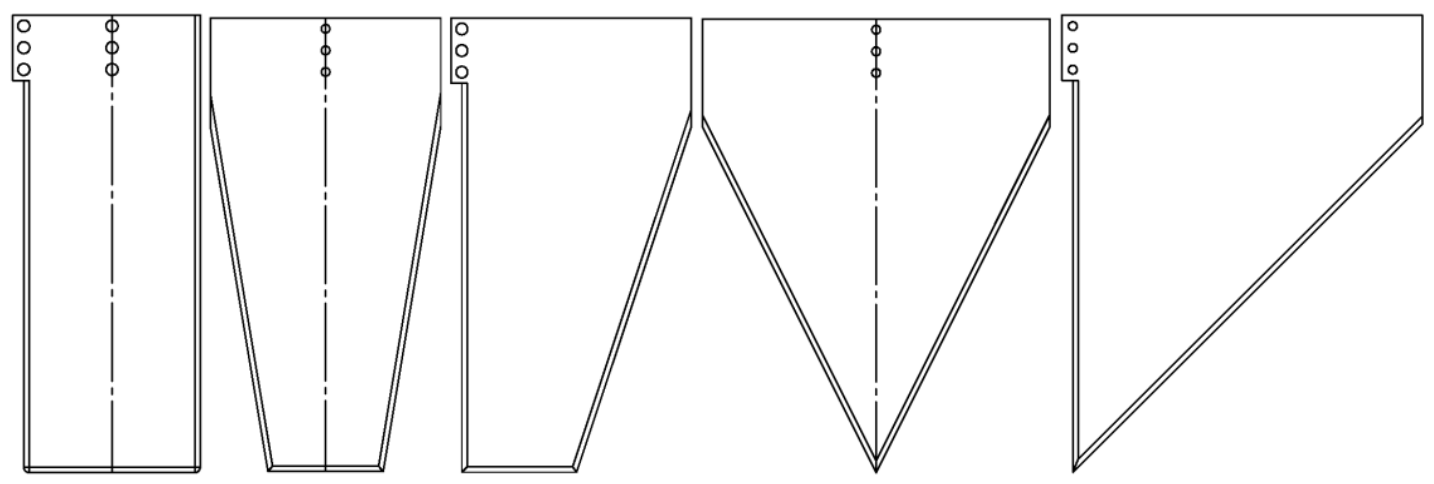

\begin{tabular}{|c|c|c|c|c|c|c|c|c|c|}
\hline \multirow{2}{*}{ Properties } & \multicolumn{2}{|c|}{ Rectangular wing } & \multicolumn{3}{|c|}{ Trapezoidal wing } & \multicolumn{3}{|c|}{ Triangular wing } & \multirow{2}{*}{$\begin{array}{c}\text { Sensor } \\
\text { adaptor \& } \\
\text { screws }\end{array}$} \\
\hline & $\mathrm{LE} / \mathrm{TE}$ & $\mathrm{ME}$ & LE & $\mathrm{TE}$ & $\mathrm{ME}$ & $\mathrm{LE}$ & $\mathrm{TE}$ & $\mathrm{ME}$ & \\
\hline Mass, $\mathrm{g}$ & \multicolumn{2}{|c|}{22.9} & \multicolumn{2}{|c|}{24.2} & 24.1 & \multicolumn{2}{|c|}{27.9} & 27.7 & 18.3 \\
\hline $\begin{array}{c}\text { Leading-edge } \\
\text { sweep angle, deg }\end{array}$ & \multicolumn{2}{|c|}{0} & 0 & 18.43 & 9.46 & 0 & 45 & 26.57 & \\
\hline Tapper ratio, $c_{t} / c_{r}$ & \multicolumn{2}{|c|}{1} & \multicolumn{3}{|c|}{0.5} & \multicolumn{3}{|c|}{0} & \\
\hline
\end{tabular}

Figure 2. Illustration of different planform wings: (from left to right) rectangular wing, two trapezoidal wings (isosceles and right), two triangular wings (isosceles and right).

Table 1 Hardware mass contribution to force transducer 


\section{B. Wing Kinematics}

The wing motion is a linear pitch ramp from $0^{\circ}$ to $45^{\circ}$ with smoothing at the beginning and the end of the pitch ramp. The wing motion is divided into five phases: start phase, pitch-up phase, hold phase, pitch-return phase, and relaxation phase. The time duration for each phase is defined in Fig. 4.

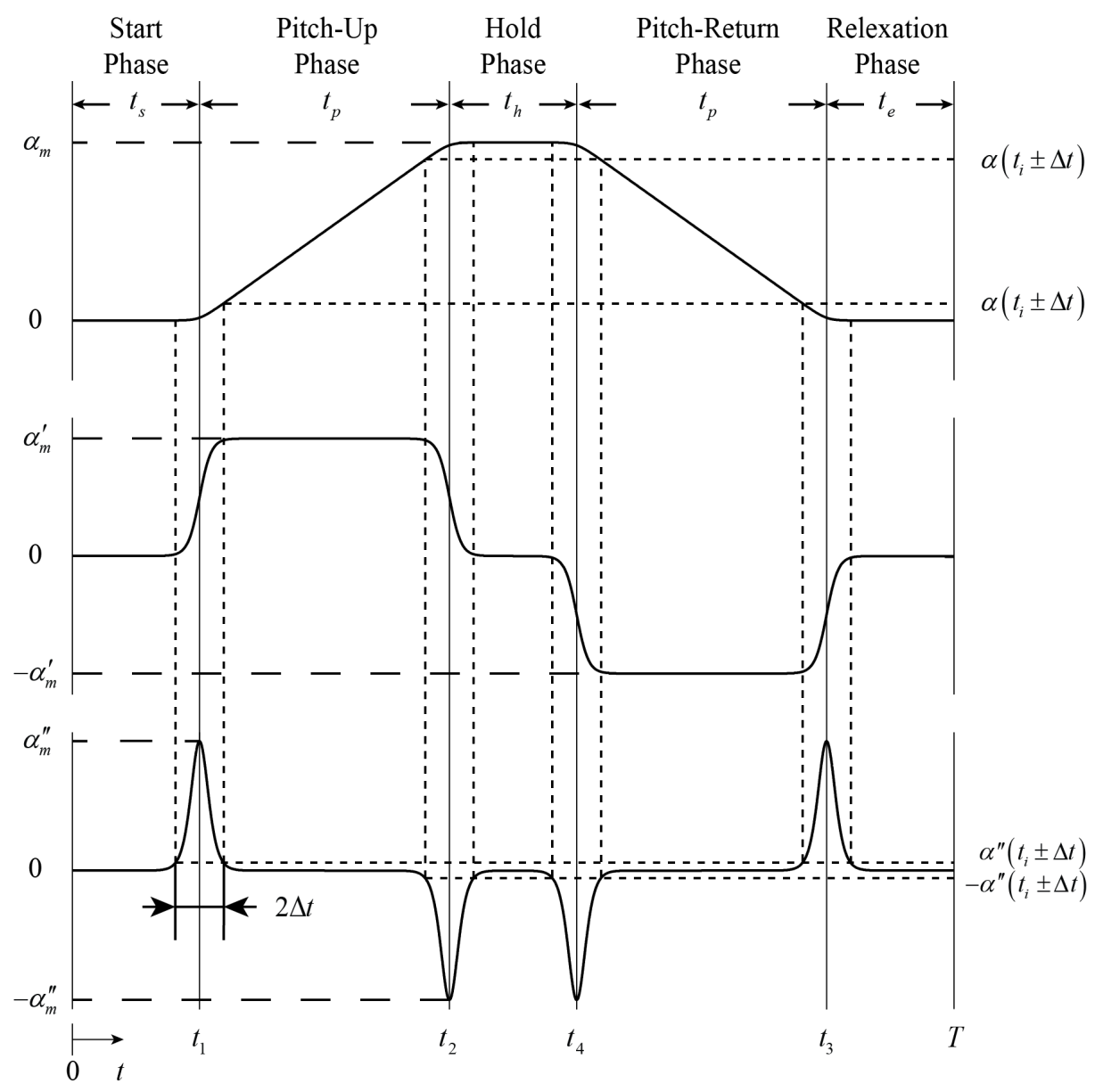

Figure 4 Illustration of linear pitch up-hold-return kinematics

The implementation of wing kinematics uses a smoothing function to minimize model vibration at the corners $\left(t_{I}\right.$ through $t_{4}$ ), defined in Eq. (5), which is a modified form of the smoothing function used by Eldredge et al [15]. The first derivative and the second derivative of the function give the motion rotation speed and acceleration, respectively. The temporal duration and pitch angle change during the smoothing regions are determined by the parameter $B$.

$$
\alpha(t)=\frac{\alpha_{m}}{2 B} \sum_{i=1}^{4}(-1)^{i+1} \ln \left\{\cosh \left[B\left(t-t_{i}\right) / t_{p}\right]\right\}, i=1,2,3,4
$$

where

$$
B=2 t_{p} / t_{a} .
$$

The parameter $B$ is the ratio of pitch time $\left(t_{p}=\alpha_{m} / \alpha_{m}^{\prime}\right)$ to pitch acceleration time $\left(t_{a}=\alpha_{m}^{\prime} / \alpha_{m}^{\prime \prime}\right)$, and is analogous to $a \alpha_{m} / 2 K$ in Eldredge's function [15] ( $a$ is the free parameter and $K$ is the reduced pitch rate) and $A \alpha_{m}$ in Yu and Bernal [19]. The duration in the pitch-up phase is the same as in the pitch-return phase; other phase durations are defined in Eq. (6) - (8).

$$
t_{s}=s t_{c}
$$




$$
\begin{aligned}
t_{h} & =h t_{c} \\
t_{e} & =e t_{c}
\end{aligned}
$$

In terms of convective times, the start parameter is $s$, the hold parameter is $h$, and the relaxation parameter is $e$. In all cases reported here the start duration is one convective time $(s=1)$. The hold duration is at least 130 convective times $(h>130)$ to ensure the flow reaching steady conditions at the maximum pitch angle of $45^{\circ}$. The relaxation duration is about 30 convective times $(e>30)$ to return the flow to the undisturbed initial condition.

The Velmex Rotary Table model B4818TS equipped with a stepper motor RK266-03 was used to implement the wing motion. The points used to implement wing motion are shown as circles in Fig. 5. There are 13 points in each smoothing transient. For all measurements the motion is repeated 60 times and phase averages are used to obtain mean values with relatively small uncertainty. Actual implementation involves a pitch up-hold-return motion similar to the kinematic used by Yu et al. [18], and Yu and Bernal [19].

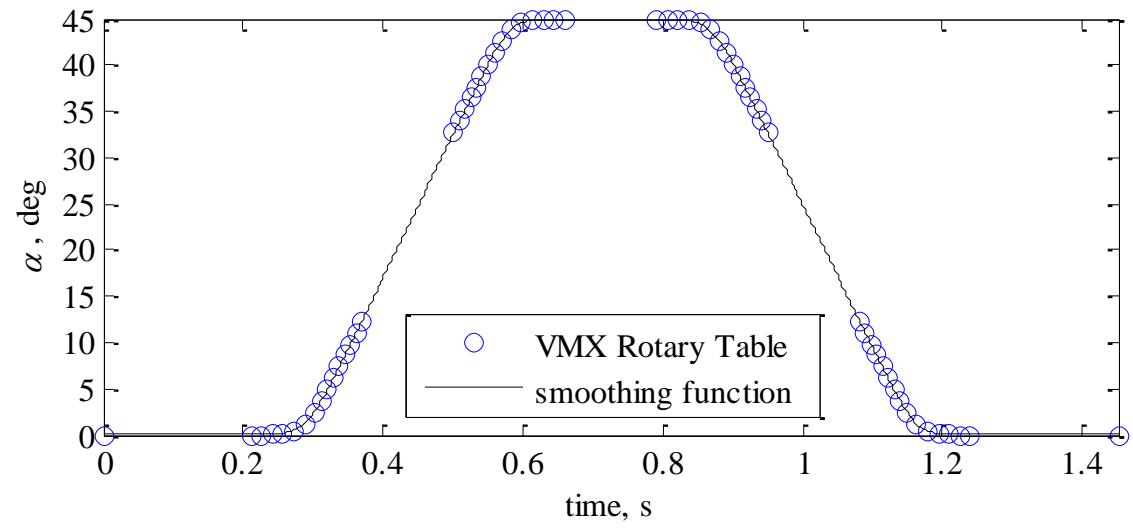

Figure 5 VMX Rotary Table kinematic implementation

\section{Test Cases for Direct Force Measurement and Data Processing}

All wings are subject to the kinematics generated using parameters listed in Table 2 with pitch rate $155 \mathrm{deg} / \mathrm{s}$ ( $S t$ $=7.0 \mathrm{k})$. The free-stream velocity used is $17.5 \mathrm{~cm} / \mathrm{s}(R e=8.9 \mathrm{k})$ and still water $(R e=0 \mathrm{k})$ which give reduced pitch rate $K=0.39$ and $K=\infty$, respectively. In terms of $S t$ - Re parameter space, the test cases are shown as square solid symbols in Fig. 6. The black square open symbols represent test cases studied in our previous work [19].

\begin{tabular}{|c|c|c|c|c|c|c|c|c|c|c|}
\hline \multirow{2}{*}{$\begin{array}{c}\alpha_{m}^{\prime}, \\
\mathrm{deg} / \mathrm{s}\end{array}$} & \multirow{2}{*}{$\begin{array}{l}\alpha_{m}, \\
\text { deg }\end{array}$} & \multirow{2}{*}{\multicolumn{2}{|c|}{$\alpha\left(t_{i} \pm \Delta t\right) / \alpha_{m} \mid \alpha^{\prime \prime}\left(t_{i}+\Delta t\right) / \alpha_{m}^{\prime \prime}$}} & \multirow{2}{*}{$\begin{array}{l}c, \\
\text { in }\end{array}$} & \multirow{2}{*}{$\begin{array}{c}\alpha_{m}^{\prime \prime}, \\
\operatorname{deg} / \mathrm{s}^{2}\end{array}$} & \multirow{2}{*}{ B } & \multicolumn{2}{|l|}{ Ref. [19] } & \multicolumn{2}{|c|}{ Ref. [15] } \\
\hline & & & & & & & A & $\mathrm{a}$ & $\mathrm{U}, \mathrm{cm} / \mathrm{s}$ & K \\
\hline 155 & 45 & $27.2 \%$ & $1 \%$ & 2 & 2937 & 11 & 14.00 & 11 & 17.5 & $\pi / 8$ \\
\hline
\end{tabular}

Table 2 Conditions to generate kinematics

Data processing of force sensor signals includes application of a low-pass filter and a tare procedure. The lowpass filter is used to remove high frequency noise associated with structural vibrations and electronic/sensor noise. The tare procedure is used to isolate the hydrodynamic force from model inertial and model weight contributions to the measured force.

A zero-phase 1st-order 2-path Butterworth filter was used to avoid phase distortion, to reduce spurious oscillations introduced by the filter, and to provide sufficient noise attenuation. The filter cutoff frequency was determined using power spectrum analysis of the motion acceleration. For the pitch rate of $155 \mathrm{deg} / \mathrm{s}$, the cutoff frequency was $8.8 \mathrm{~Hz}$ to retain $90 \%$ of the frequency content of the motion acceleration, and the corresponding attenuation at the frequency of maximum energy content is $-5.39 \mathrm{~dB}$. It should be noted that the noise introduced by the rotary stage stepper motor is in the range of $100 \mathrm{~Hz}$ to $600 \mathrm{~Hz}$, and the wing vibration fundamental frequencies in 
air and in still water were determined by comparing with the configuration without wing and is also significantly higher than the filter cutoff frequency.

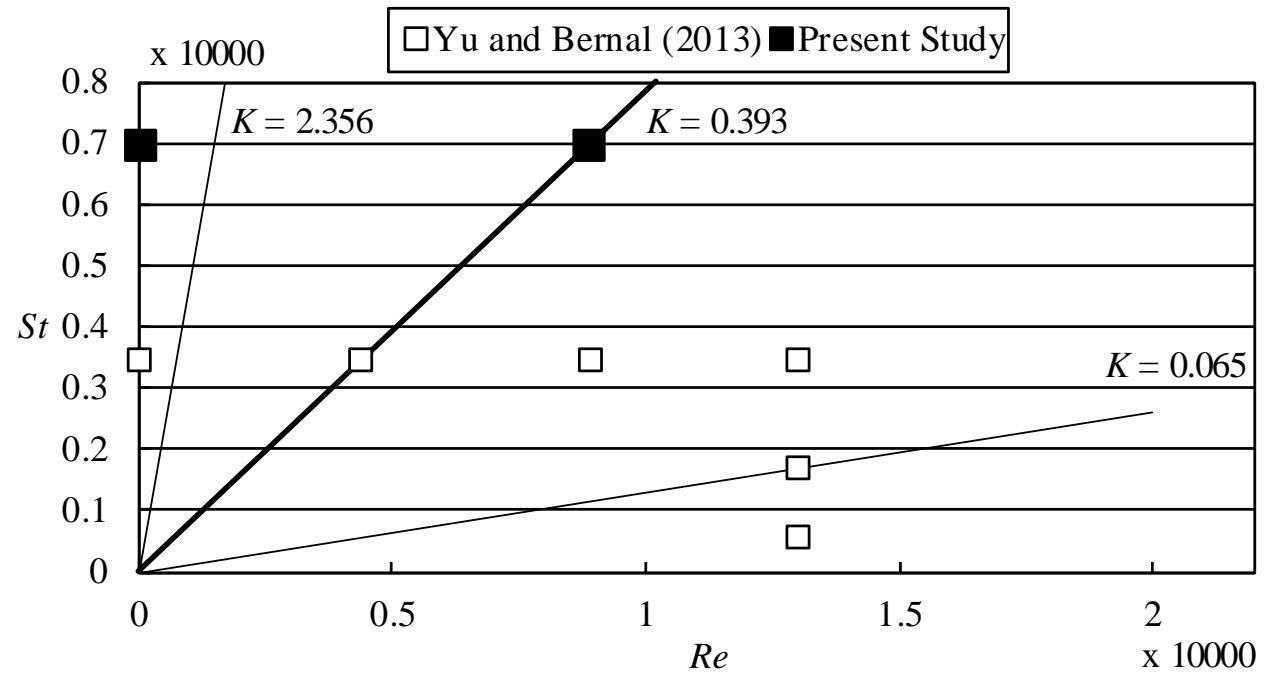

Figure 6 Test cases in Stokes number $(S t)$ and Reynolds number $(\boldsymbol{R e})$ parameter space. The red square solid symbols represent test cases in current study; the black square open symbols represent test cases in [19]

Tare procedures are generally necessary to remove inertia and weight contributions to the measured force due to the wing acceleration and position. These include static tares and dynamic tares. The static tares are measurements in air and still water at fixed angle from $0^{\circ}$ to maximum angle of attack $45^{\circ}$ with an increment of $3^{\circ}$. The dynamic tares are measurements in air with the same kinematics as in the flow experiments. In addition, the force measurements at fixed angle of attack in steady flow are also considered. The kinematics is similar to static tare measurements but with longer hold duration at the free-stream velocity of interest. In all force measurements the same filter cutoff frequency was used.

All measurements are repeated 60 times from $0^{\circ}$ to fixed angle of attack with sampling rate of $5000 \mathrm{~Hz}$, and ensemble averages are reported. It was found that the present wing configuration yields negligible static tares because of the very small mass of the wing and mounting hardware.

\section{Flow Visualization}

The flow visualization was conducted using a dye rake $(7$ probes with 1" spacing), a camera, two syringe pumps, and two different color food dyes (i.e. blue and red). Fig. 7 is an illustration of wing configuration used for dye visualization, a background board with thickness of 0.24 " was employed to exclude distracting background features and reflection from the water surface. The injection rake was placed at $50 \%$ of wing span for all wing planforms, as shown by the red line in Fig. 7, and 3 mean chords upstream of the leading edge. Since the density of the dyes (ESCO Foods) is $1012 \mathrm{~kg} / \mathrm{m}^{3}$, they were mixed with alcohol to match the water density, $998 \mathrm{~kg} / \mathrm{m}^{3}$. The

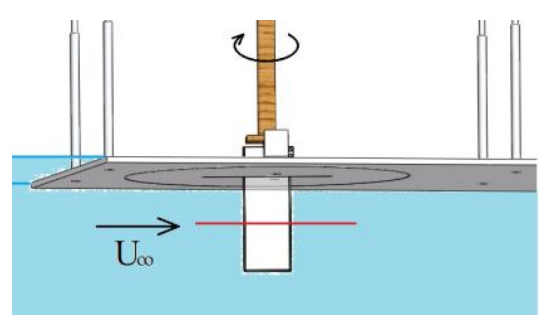

Figure 7 Wing configurations in flow visualization

images were recorded using Nikon D3100 camera at a frame rate of $30 \mathrm{~Hz}$. Images of the side view and top view are reported. The side view provides the field of view of flow about the wing chord; the top view gives the field of view of flow about the wing span.

\section{Results and Discussion}

This section reports force data examined in terms of convective time, pitching time, and angle of attack. Theoretical results derived using lifting-line theory and including pitch rate effects [23] are also compared with the 
measurements. Images from flow visualization are discussed to relate the flow development and aerodynamic forces.

\section{A. Lifting-Line theory}

Theoretical estimation of pitch rate effects is derived based on linear airfoil theory [23] and lifting-line theory. The normal velocity is given in Eq. (9) for 2D airfoil at any instant time.

$$
U_{\infty, n}(x, t)-U_{\Omega, n}(x, t)+w^{\prime}(s, t)=0
$$

The first term is the normal velocity from the free-stream, the second term is from the pitch motion, and the third term is from the wing bound vorticity. In sequence, they are formulated in Eq. (10) - (12), respectively, assuming small camberline slope $(d z / d x)$ and $z(x) / x<<1$ [22].

$$
\begin{gathered}
U_{\infty, n}(x, t)=U_{\infty}(\alpha(t)-d z / d x) \\
U_{\Omega, n}(x, t)=\alpha^{\prime}(t)\left(x_{p}-x\right) \\
w^{\prime}(s, t)=\frac{1}{2 \pi} \int_{0}^{\pi} \frac{\gamma(\theta, t) \sin \theta}{\cos \theta-\cos \theta_{0}} d \theta
\end{gathered}
$$

Introducing the solution for the strength of a vortex sheet given in Eq. (13), the effective camberline, sectional lift coefficient and sectional pitch moment are obtained and given in Eq. (14) - (16) for fixed angle of attack, respectively. Since steady Bernouli equation is assumed to evaluate the lift coefficient, and the pitch moment coefficient, the results are valid for steady flow conditions. Also non-circulatory effects and normal velocity from vorticity in the wake are not included.

$$
\begin{gathered}
\gamma(\theta)=2 U_{\infty}\left[A_{0} \frac{1+\cos \theta}{\sin \theta}+\sum_{n=1}^{\infty} A_{n} \sin n \theta\right] \\
(d z / d x)_{e f f}=d z / d x+\alpha_{m}^{\prime}\left(x_{p}-x\right) / U_{\infty}=\alpha-A_{0}+\sum_{n=1}^{\infty} A_{n} \cos n \theta_{0} \\
c_{l}=\frac{2 \rho U_{\infty} \Gamma}{\rho U_{\infty}^{2} S}=\pi\left[2 A_{0}+A_{1}\right] \\
c_{m, L E}=-\frac{\pi}{2}\left[A_{0}+A_{1}-\frac{A_{2}}{2}\right]
\end{gathered}
$$

where coefficient $A_{0}$ and $A_{n}$ are found using Eq. (17) and Eq. (18), respectively.

$$
\begin{gathered}
A_{0}=\alpha-\frac{1}{\pi} \int_{0}^{\pi}(d z / d x) d \theta_{0}+\frac{\alpha_{m}^{\prime} c}{2 U_{\infty}} \cos \theta_{p} \\
A_{n}=\frac{2}{\pi} \int_{0}^{\pi}(d z / d x) \cos n \theta_{0} d \theta_{0}+\frac{\alpha_{m}^{\prime} c}{2 U_{\infty}}
\end{gathered}
$$

In our experiments the wings are all flat plates, the corresponding sectional lift and pitch moment coefficients are given in Eq. (19) and Eq. (20), respectively.

$$
\begin{gathered}
c_{l}=a_{0}\left(\alpha-\alpha_{L=0}\right)=2 \pi\left[\alpha+K\left(\cos \theta_{p}+\frac{1}{2}\right)\right] \\
c_{m, L E}=-\frac{\pi}{2}\left[\alpha+K\left(\cos \theta_{p}+1\right)\right]
\end{gathered}
$$

Considering now a finite aspect ratio wing, the lift and drag coefficients are found by introducing the slope of lift coefficient curve $\left(a_{0}\right)$ and the zero lift angle of attack $\left(\alpha_{L=0}\right)$ from 2D airfoil theory, as shown in Eq. (19). The angle of attack, including tip vortex effects, is given by Eq. (21) to determine the coefficients $D_{n}$. The first term on the 
right-hand side represents the induced angle of attack, and the last two terms represent the effective angle of attack [22].

$$
\alpha(\phi)=\sum_{n=1}^{N} n D_{n} \frac{\sin n \phi}{\sin \phi}+\frac{4 b}{a_{0} c(\theta)} \sum_{n=1}^{N} D_{n} \sin n \phi-K\left(\cos \theta_{p}+\frac{1}{2}\right)
$$

Hence, the lift and drag coefficients are then given by Eq. (22) and (23), respectively, and are shown as green curves in Fig. (14) - (15) to represent the theoretical prediction for a rectangular wing discussed later.

$$
\begin{gathered}
C_{L}=\frac{2}{\rho U_{\infty}^{2} S} \int_{-b / 2}^{b / 2} \rho U_{\infty} \Gamma(y) d y=\frac{b^{2}}{S} \pi D_{1} \\
C_{D_{i}}=\frac{2}{\rho U_{\infty}^{2} S} \int_{-b / 2}^{b / 2} \rho U_{\infty} \Gamma(y) \alpha_{i}(y) d y=\frac{b^{2}}{S} \pi \sum_{n=1}^{N} n D_{n}^{2}
\end{gathered}
$$

\section{B. Steady Flow Measurements $K=0$}

Figures 8 - 9 show the wing planform effects in the steady flow, which was measured at fixed angle from $3^{\circ}$ to $45^{\circ}$ with an increment of $3^{\circ}$ in the flow field with $U_{\infty}=17.5 \mathrm{~cm} / \mathrm{s}$ (i.e. $R e=8.9 \mathrm{k}$ ). Each fixed angle was repeated 60 times from initial angle of attack $0^{\circ}$; all data are processed using the same filter and cutoff frequency as in cases with $K=0.39$. The mean and standard deviation are evaluated after the flow reached steady state (i.e. after 80 convective times), and the sample duration is 50 convective times. The lifting-line theory results for a rectangular wing are also shown in figures.
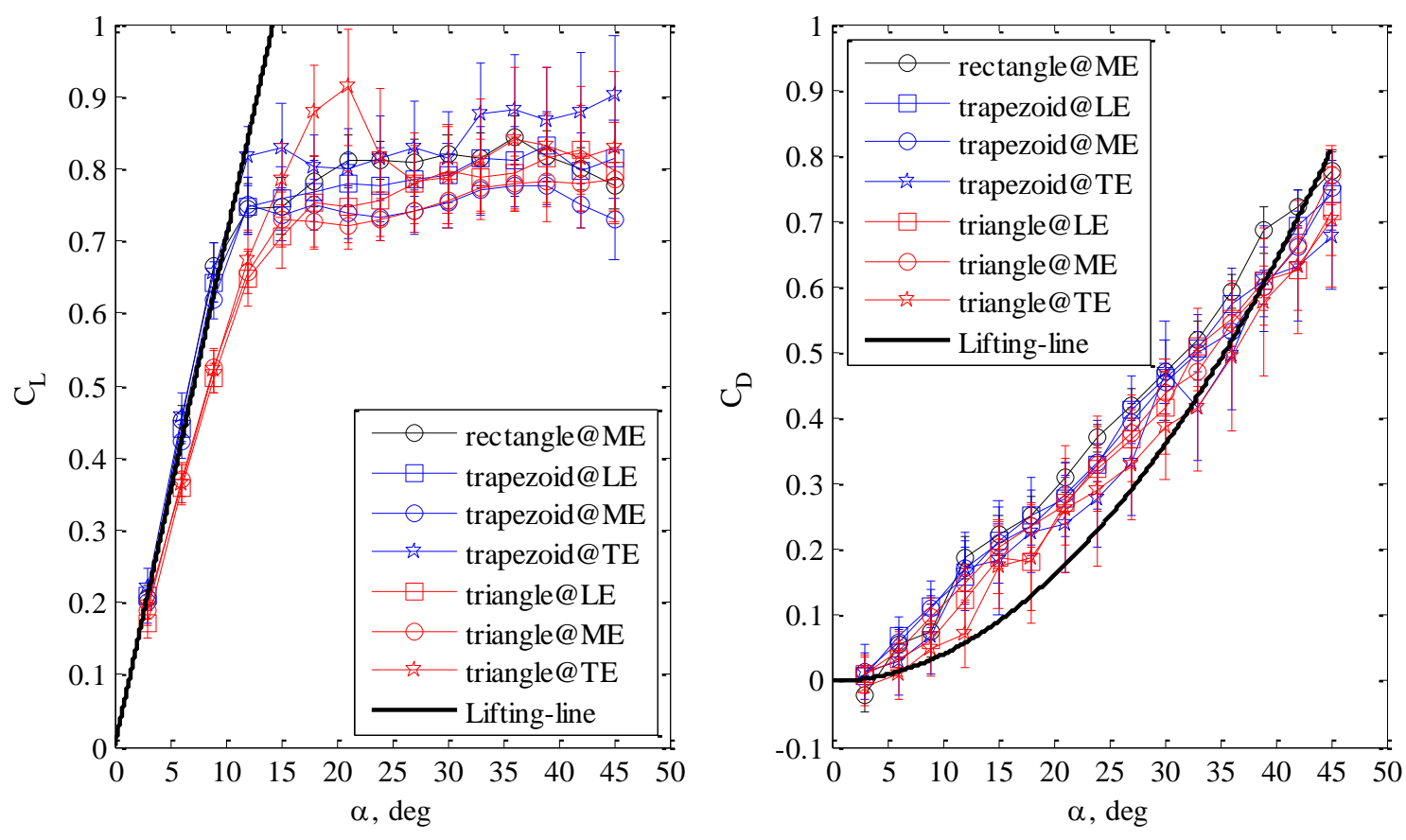

\section{Figure 8 Force coefficients as a function of angle of attack for (left) lift coefficient and (right) drag coefficient}

Figure 8 gives lift coefficient in the left panel and drag coefficient in the right panel. Two main features are observed. The first is, for rectangular and trapezoidal wings (i.e. tapper ratio higher than 0.5) the lift coefficients follow the lifting-line theory up to $9^{\circ}$. Lower tapper ratio wing (i.e. triangular wing) gives lower lift coefficient. The second is that trapezoidal and triangular wings for trailing edge pivot stall at a higher angle of attack of $15^{\circ}$ and $21^{\circ}$, respectively. For drag coefficient in the right hand side, tapper ratio effects are small, drag coefficients increase linearly with angle of attack, which is consistent with lifting-line theory. 
Pitching moment coefficients about the pivot axis as a function of angle of attack are shown in the left hand side of Fig. 9. Leading edge pivot gives negative pitching moment coefficient, whereas mid-chord and trailing edge pivot produce positive pitching moment coefficients. At lower angle of attack, effects of tapper ratio are small. The triangular wing with trailing edge pivot stalls at $21^{\circ}$, which is consistent with the observation from the lift coefficient in Fig. 8. In addition, the large fluctuations at post-stall angles shown in the lift coefficient are not observed in the pitching moment coefficient.

Location of center of pressure as a function of angle of attack is shown in the right hand side of Fig. 9, which is evaluated using Eq. (24) and normalized with the wing mean chord. For tapper ratio higher than 0.5, the center of pressure is at quarter chord at lower angle of attack, and moves to about $40 \%$ of wing chord for post stall condition. For triangular wing shown as red symbols, different profile for each pivot axis is obtained, which is possibly due to three-dimensional effects.

$$
x_{c p}=x_{p}-M_{p} / N
$$
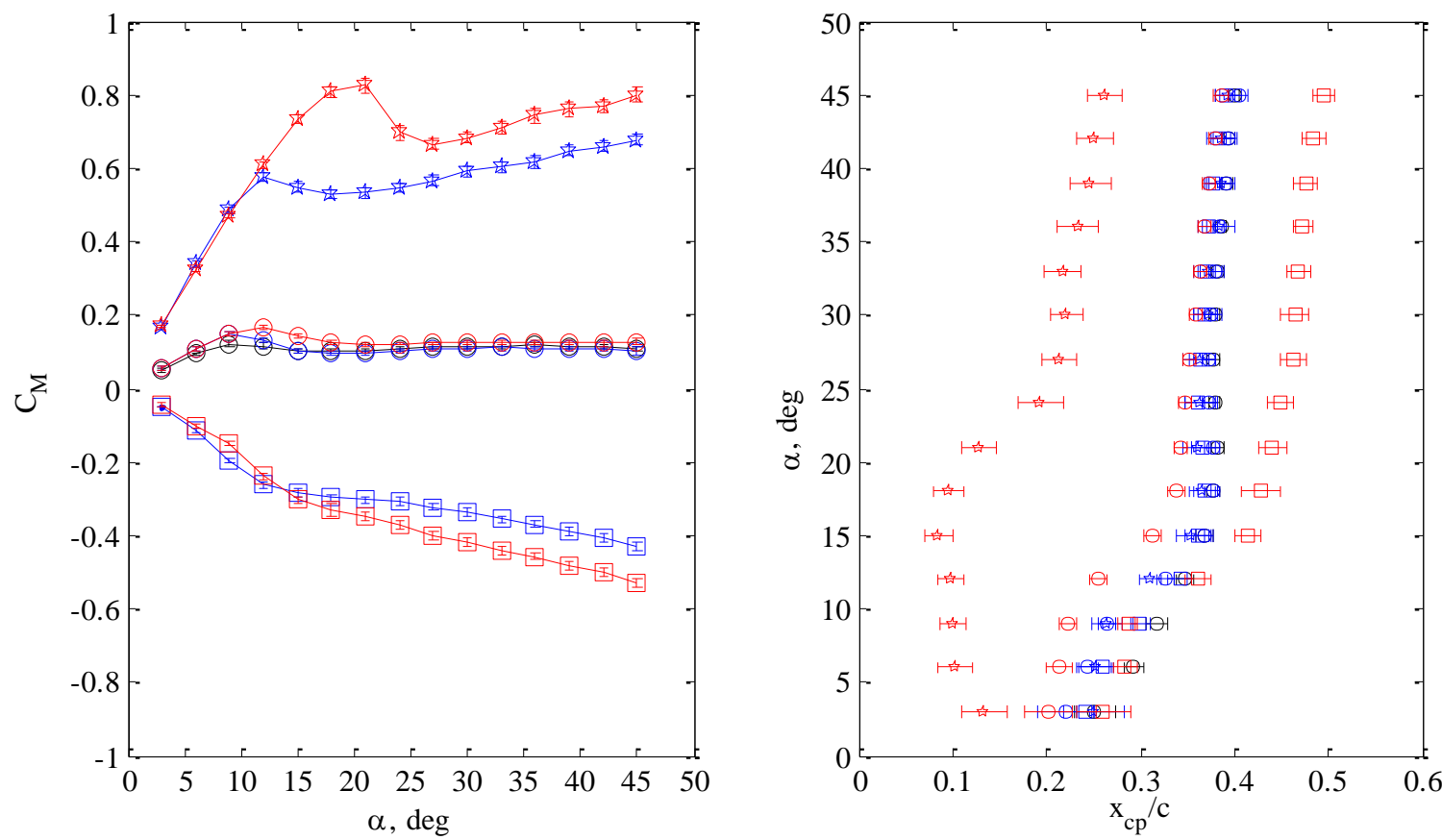

Figure 9 (left) pitching moment evolution as a function of angle of attack and (right) center of pressure as a function of angle of attack. Square, circle, star symbols represent pivot axes at leading edge, mid-chord, and trailing edge, respectively. The black, blue, and red colors represent rectangular wing, trapezoidal wing, and triangular wing, respectively.

\section{Axial Force and Normal Force Evolution as a function of Convective Time}

Figures $10-12$ present the normal and axial forces evolution as a function of convective time for leading edge pivot axis (LE), mid-chord pivot axis (ME), and trailing edge pivot axis (LE), respectively. The black, blue, and red curves present the rectangular, trapezoidal, and triangular wings, respectively. The solid and dotted curves are measurements in $R e=8.9 \mathrm{k}$ and still water, respectively. The wing angular position is presented as dashed curve. The time scale at the bottom starts from the first corner $t_{l}$, as denoted in Fig. 4, and the time scale on the top starts when motion stops at $45^{\circ}$ for flow visualization during the hold-phase. Error bars are the standard deviation computed using over 60 samples and depend on free-stream velocity and pivot axis location. The standard deviation is small and of the order of $20 \mathrm{mN}$, they are very small compared to the magnitude of the normal force fluctuations but of the order of the axial force fluctuations. The vertical gray lines are selected phases for which flow visualization results are provided in the appendix. 

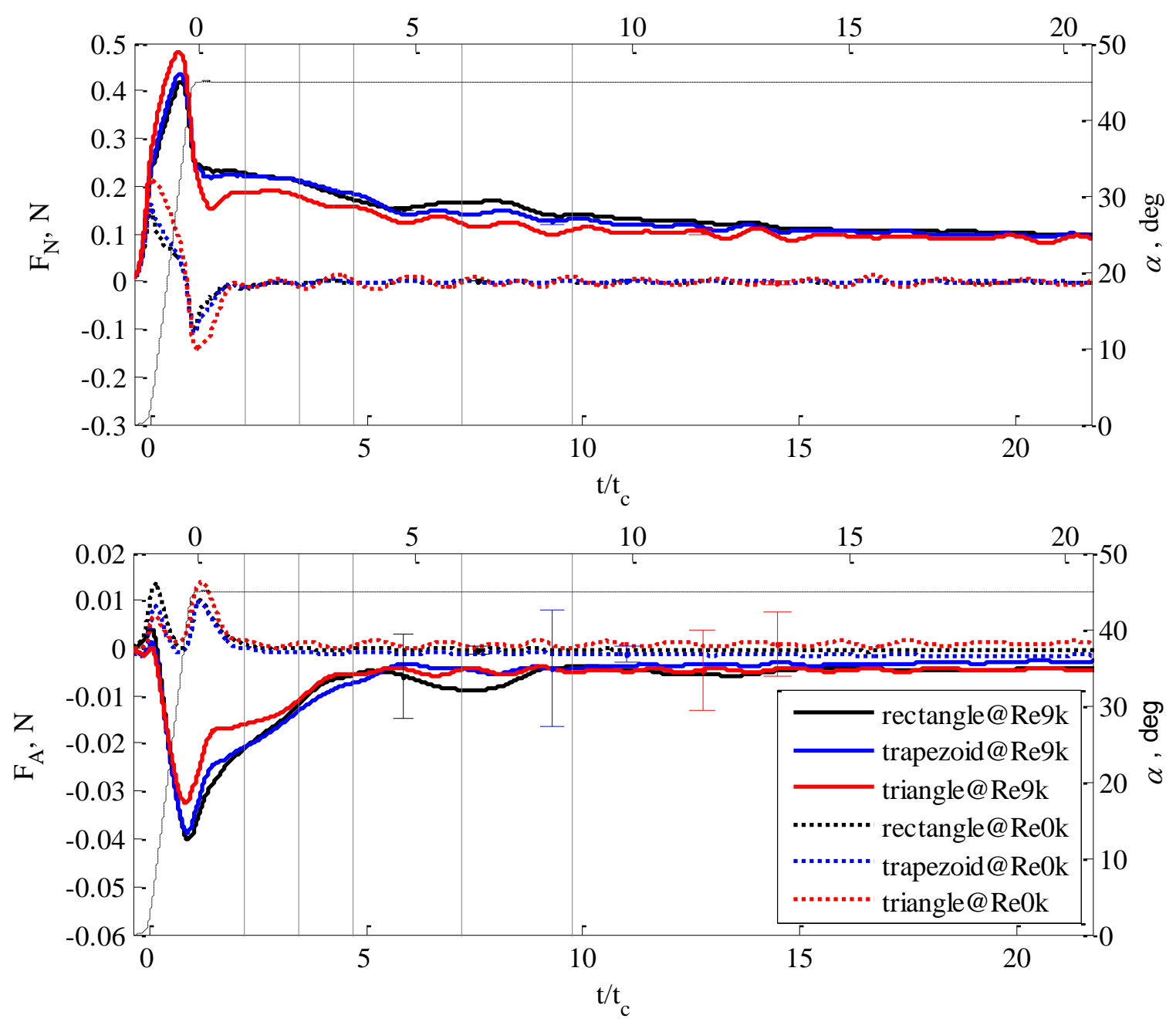

Figure 10 Force evolutions as a function of convective time at leading edge pivot axis in (top) normal force and (bottom) axial force

Two common features are observed for different wing planforms and pivot axis. First, normal force increases rapidly during the pitch-up phase, and decreases gradually during the hold-phase at $45^{\circ}$ to reach steady state after approximately 30 convective times, where tapper ratio effects are small. Secondly, the oscillatory normal force evolution is observed after wing reaches maximum angle of attack (i.e. $45^{\circ}$ ) and before steady state condition is reached. For higher tapper ratio wings (i.e. rectangular wing and trapezoidal wing), oscillation period is about 5 times convective times, which is in phase with axial force. For the triangular wing and LE pivot, small amplitude oscillations are found along the normal force and independent of free-stream velocity, which is due to much stronger surface waves generated during the pitching motion. This oscillatory behavior is also observed for TE pivot in still water but not for ME pivot. In addition, all axial forces subject to free-stream velocity are negative and significant smaller than normal forces, which indicates occurrence of stronger leading edge suction. In the still water, axial forces are positive at LE and negative at TE, corresponding to the phases of motion acceleration. Neutral axial forces are observed at ME pivot. 

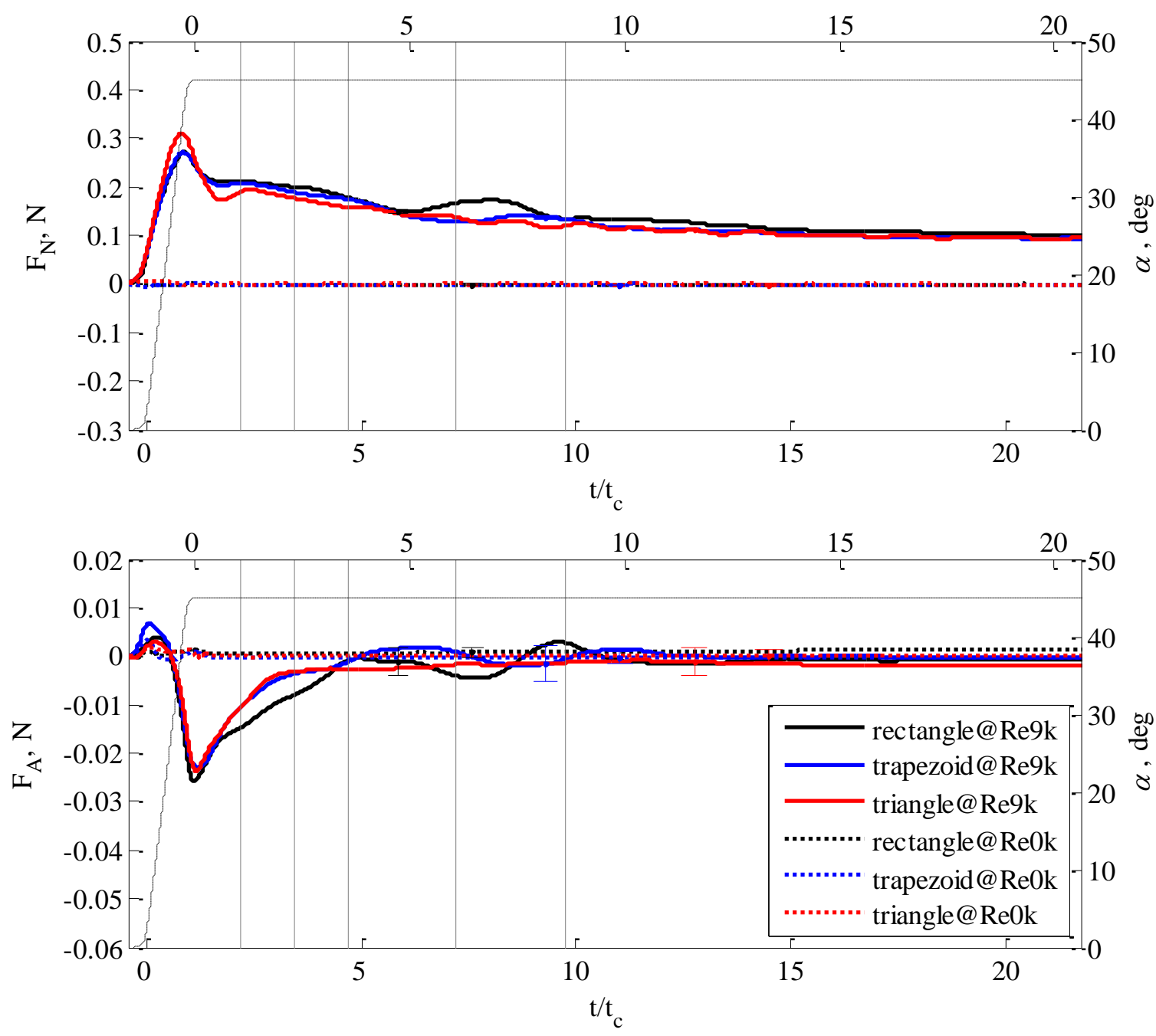

Figure 11 Force evolutions as a function of convective time at mid-chord pivot axis in (top) normal force and (bottom) axial force

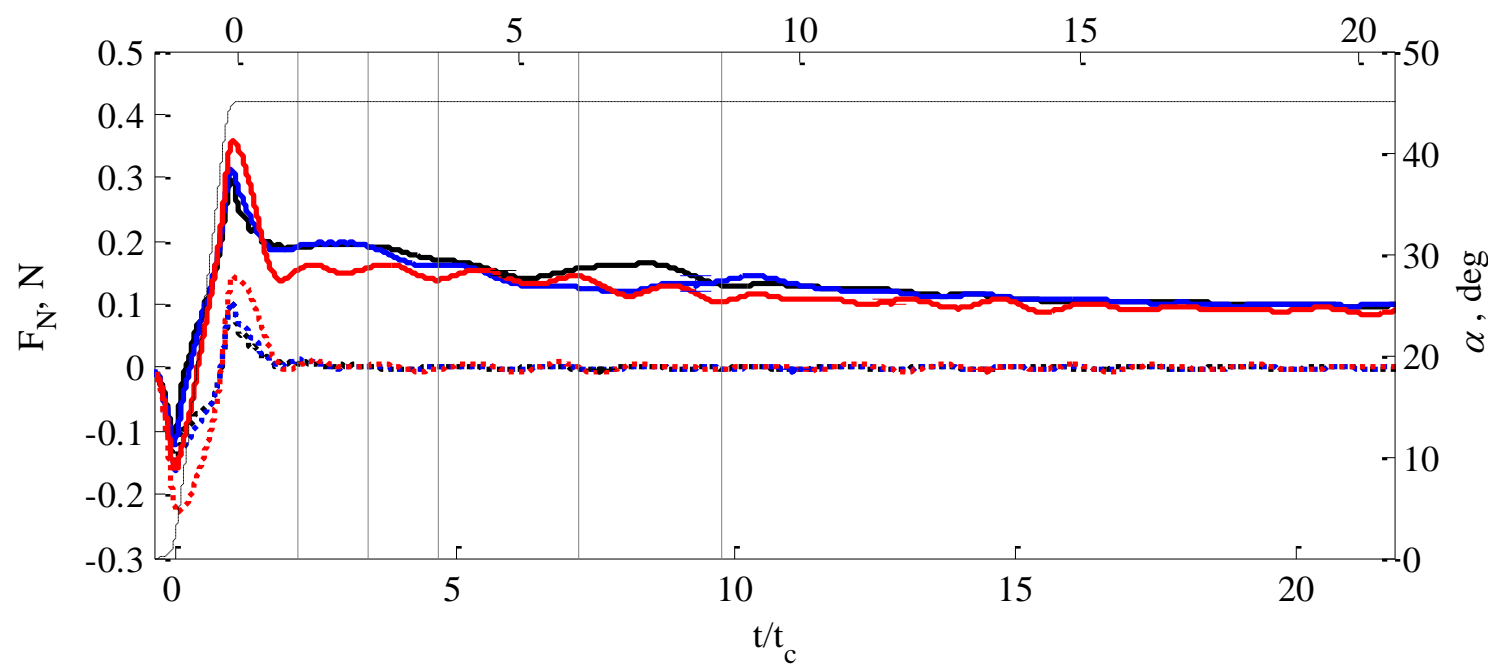




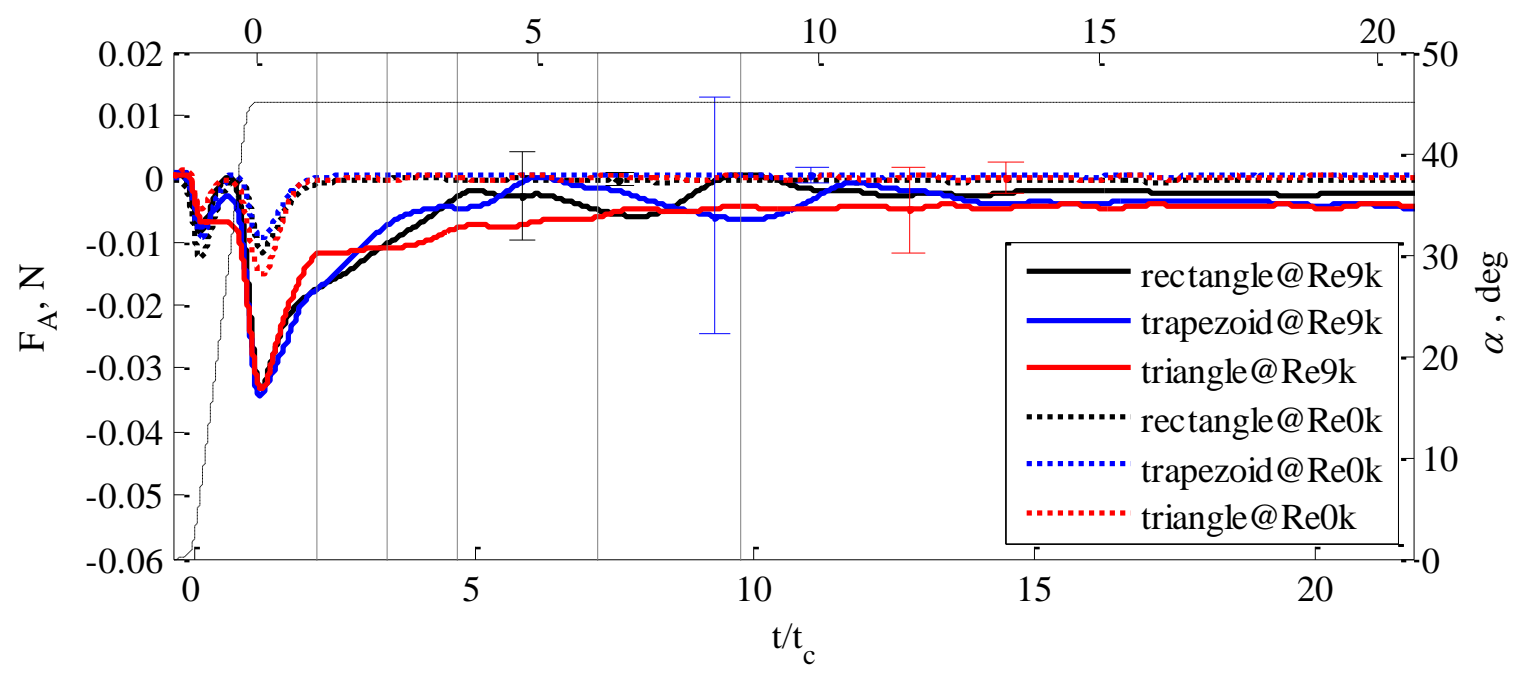

Figure 12 Force evolutions as a function of convective time at trailing edge pivot axis in (top) normal force and (bottom) axial force

\section{Effects of Pivot Axis on Normal Force}

Figures 13 - 15 shows normal force evolutions as a function of pitch time for rectangular, trapezoidal, and triangular wings, respectively. The blue, black, and red curves represent pivot axis at leading edge, mid-chord, and trailing edge, respectively. The solid and dotted curves are for $K=0.39$ (Re $=8.9 \mathrm{k}$ ) and $K=\infty$ (still water), respectively. The motion acceleration is given as a black dashed curve.

At a given pivot axis all wing planforms show similar trends in force development. For leading edge pivot, the normal force spike is positive at the beginning of pitching motion and negative at the end of pitching motion, which is consistent with the direction of the motion acceleration. However, for trailing edge pivot axis, the direction of pitching motion is in the opposite direction of the normal force, as a result, the negative normal force spike is observed at beginning of pitching motion and positive at the end of pitching motion. For mid-chord pivot, normal force spikes are not observed, which indicates non-circulatory apparent mass effects are not present. Additionally, as tapper ratio decreases, the force evolution is smoother, even in the still water measurement, which indicates stronger three dimensional rotation rate effects. Additionally, measurements for leading edge pivot and trailing edge pivot in still water give symmetrical force evolution and zero force at mid-chord pivot as expected, which give a good indication of the accuracy of the measurements.

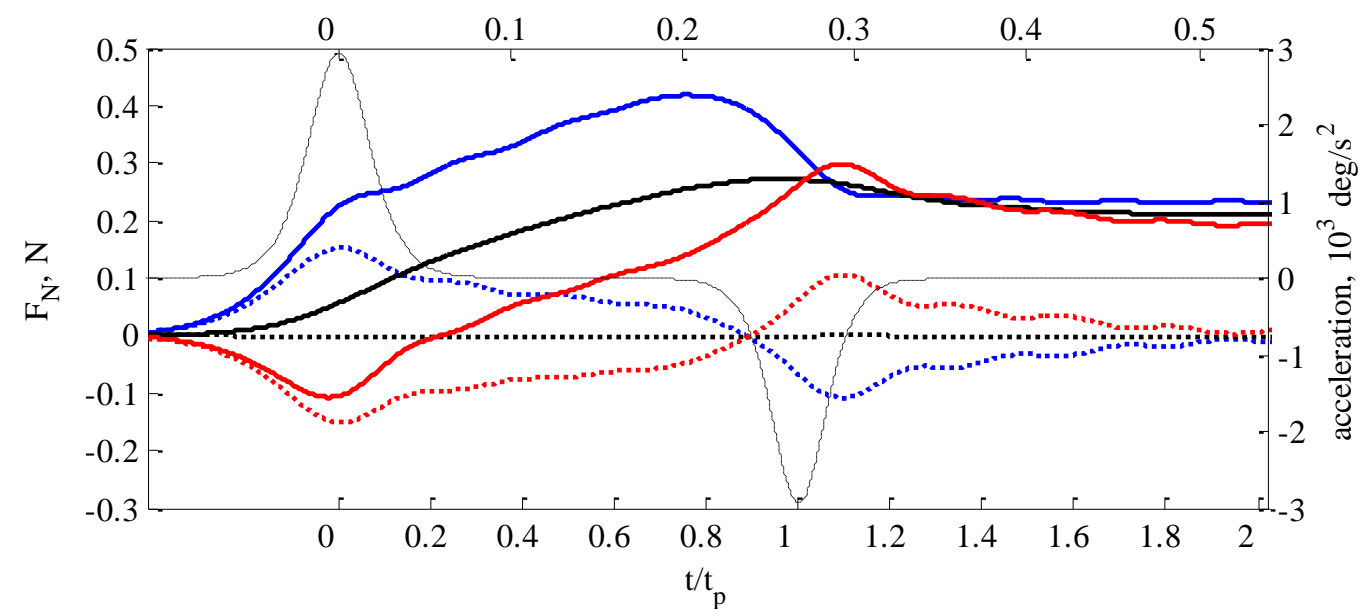

Figure 13 Normal force evolutions as function of pitch time for rectangular wing. Blue, black, and red curves represent the pivot axis at leading edge, mid-chord, and trailing edge, respectively. The solid curves are for $K=$ 0.39 , and the dotted curves are $K=\infty$ (still water) 


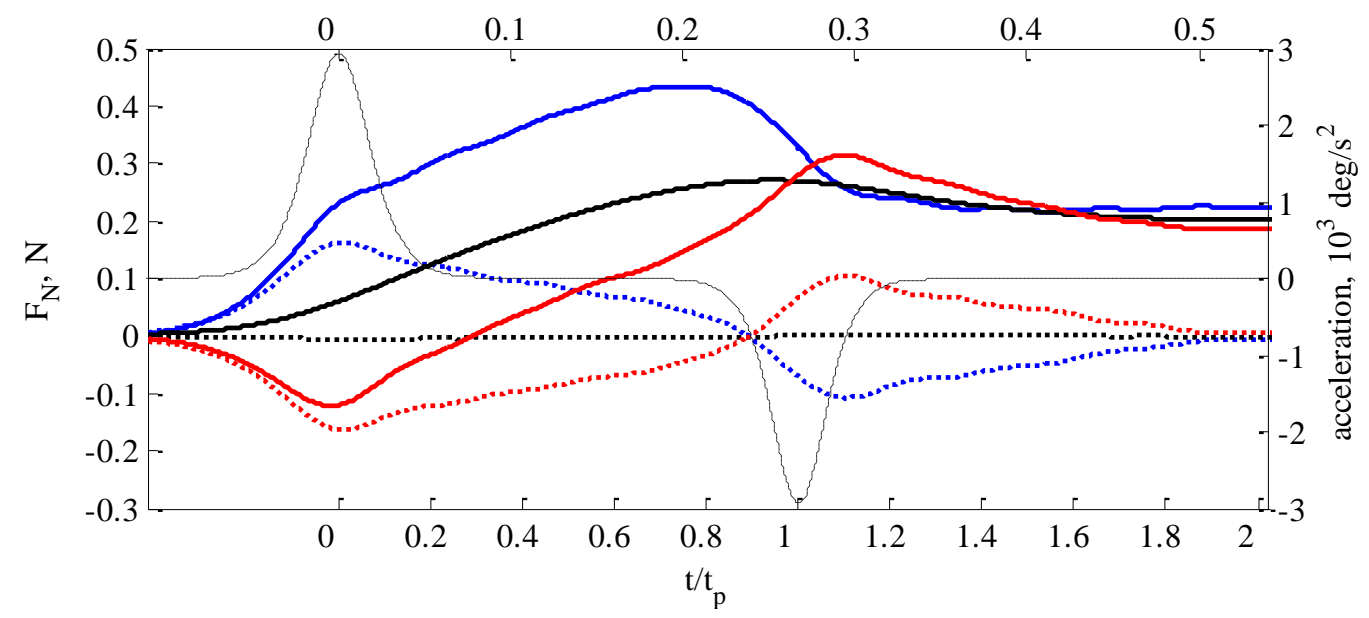

Figure 14 Normal force evolutions as function of pitch time for trapezoidal wing. Blue, black, and red curves represent the pivot axis at leading edge, mid-chord, and trailing edge, respectively. The solid curves are for $K=$ 0.39 , and the dotted curves are $K=\infty$ (still water)

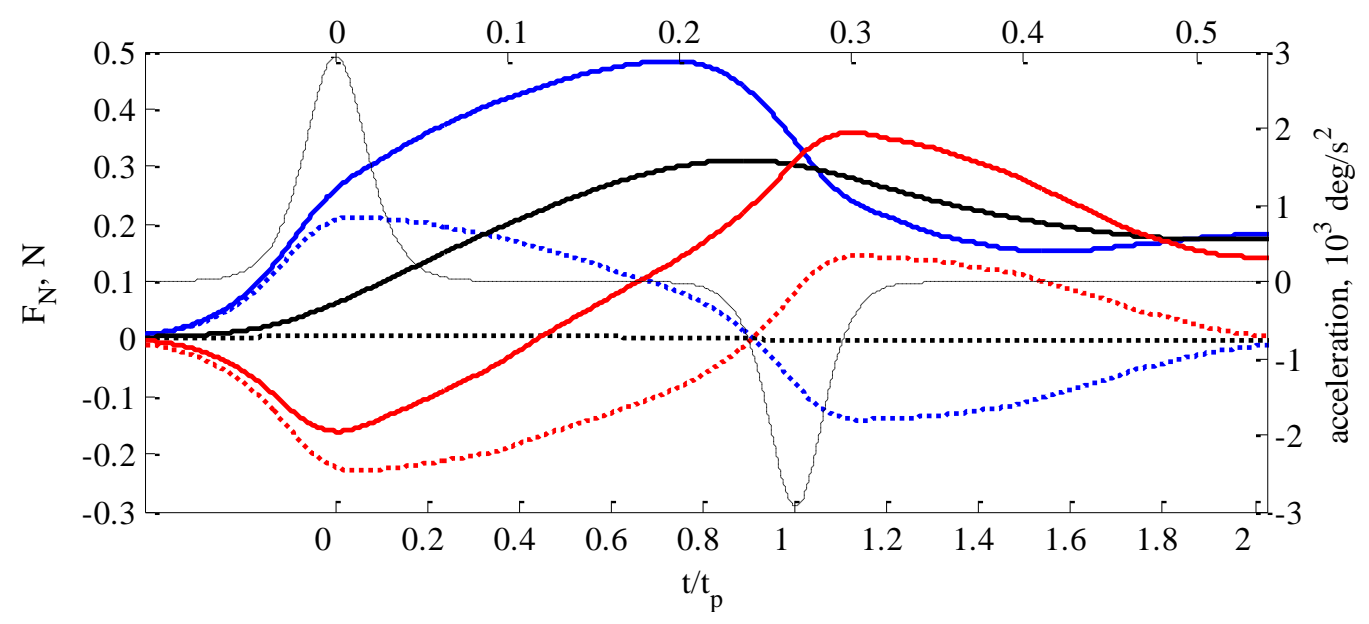

Figure 15 Normal force evolutions as function of pitch time for triangular wing. Blue, black, and red curves represent the pivot axis at leading edge, mid-chord, and trailing edge, respectively. The solid curves are for $K=$ 0.39 , and the dotted curves are $K=\infty$ (still water)

\section{E. Effects of Wing Planform on Force Coefficients}

Figures $16-17$ shows force evolutions as a function of angle of attack for leading edge pivot, mid-chord pivot, and trailing edge pivot, respectively. The black, blue, and red curves represent rectangular, trapezoidal, and triangular wing, respectively. The solid curves are for cases $K=0.39$ and dotted curves are for cases $K=0$ (denoted as steady). The theoretical results from lifting-line theory for rectangular wings are plotted as green curves.

Figure 16 shows lift and drag coefficients versus angle of attack at leading edge pivot axis. For lift coefficient at $K=0.39$, non-circulatory apparent mass effects are observed at the beginning and the end of pitch-up phase. During the constant pitch rate phase, lower tapper ratio wings produce higher lift and drag coefficients, well beyond the prediction by lifting-line theory and steady flow measurements, which are due to three-dimensional rotation rate effects. The evolutions of lift coefficient for triangular wings do not increase linearly with angle of attack. For the drag coefficient, the pitch rate effects are over-predicted by lifting-line theory at lower angle of attack and underestimated at higher angle of attack. 

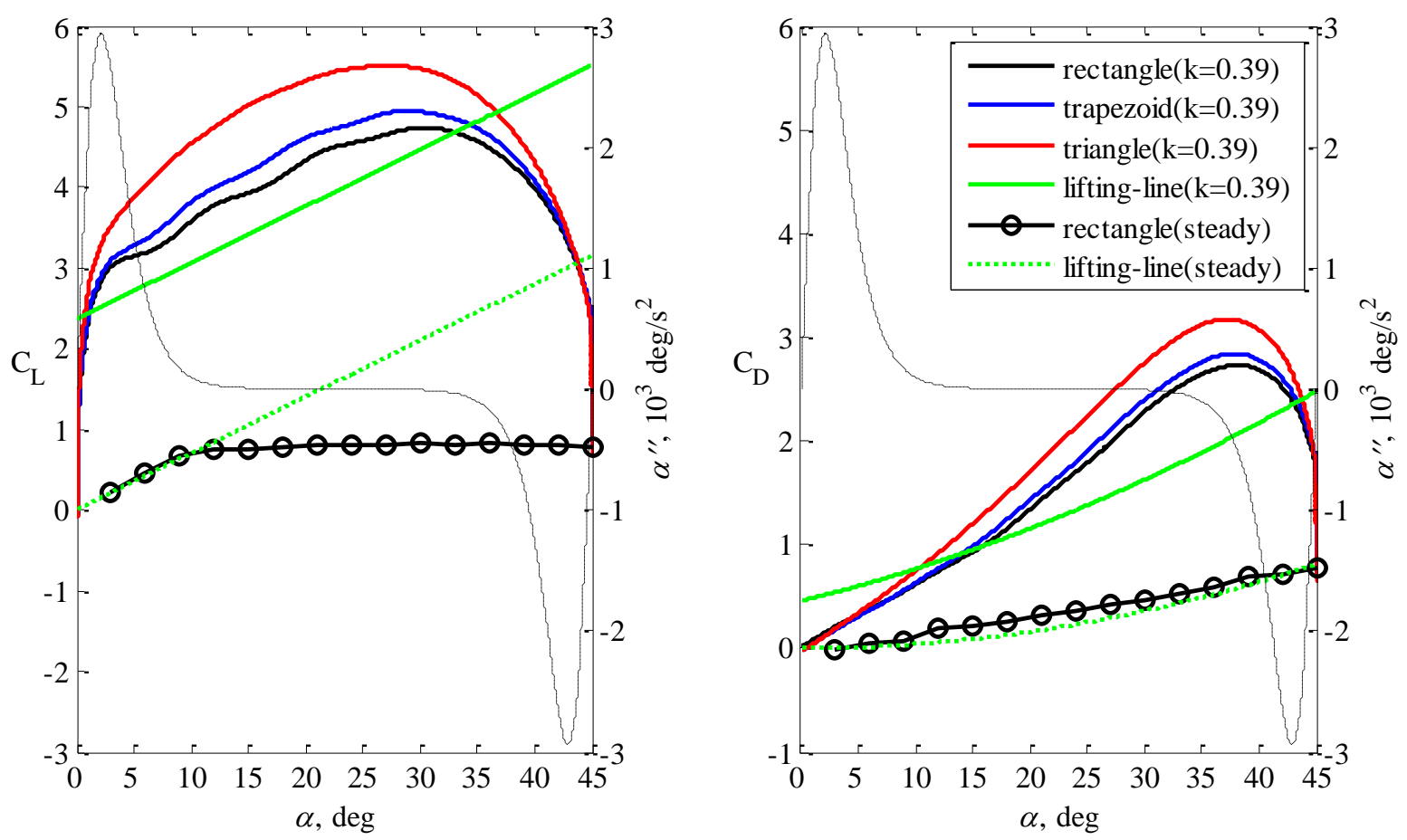

Figure 16 Force evolutions as a function of angle of attack at leading edge pivot axis for (left) lift coefficient and (right) drag coefficient

For mid-chord pivot, as shown in Fig. 17, non-circulatory apparent mass effects are absent. Higher tapper ratio wing (i.e. $\geq 0.5$ ) gives approximately the same lift and drag coefficients, the wing with lower tapper ratio gives higher lift and drag coefficients at higher angle of attack, which do not follow the theoretical result. For all wing planforms, forces are higher than steady flow measurement for a rectangular wing.

For trailing edge pivot, as shown in Fig. 18, non-circulatory apparent mass effects are found at the beginning and the end of pitching motion. Lift coefficients for tapper ratio higher than 0.5 during the constant pitch rate phase follow the theoretical estimation closely, whereas drag coefficients are not well predicted. At lower angle of attack, lift and drag coefficients are below the steady flow measurement for rectangular wing and higher at larger angle of attack.

Figure 19 shows the pitching moment coefficients about the pivot axis for different wing planforms, the black, blue, and red curves represent rectangular, trapezoidal, and triangular wings, respectively. The steady flow measurement for rectangular wing at mid-chord is plotted as circle symbols, and evaluated for leading edge pivot and trailing edge pivot. As pivot axis at leading edge, negative pitching moments are found in the range of pitch angle, which are consistent with steady flow measurements about corresponding pivot axis. Lower tapper ratio gives higher pitching moment coefficient. For mid-chord pivot, higher tapper ratio wings ( $\geq 0.5$ ) give coincident pitching moment coefficients, lower the tapper ratio gives higher pitching moment coefficients, all of them have magnitude less than one. Moreover, negative pitching moment coefficient is observed at lower angle of attack, which is contrary to the steady flow measurements. For trailing edge pivot, negative pitching moment coefficients are also observed at lower angle of attack with much larger amplitude than for mid-chord pivot. 

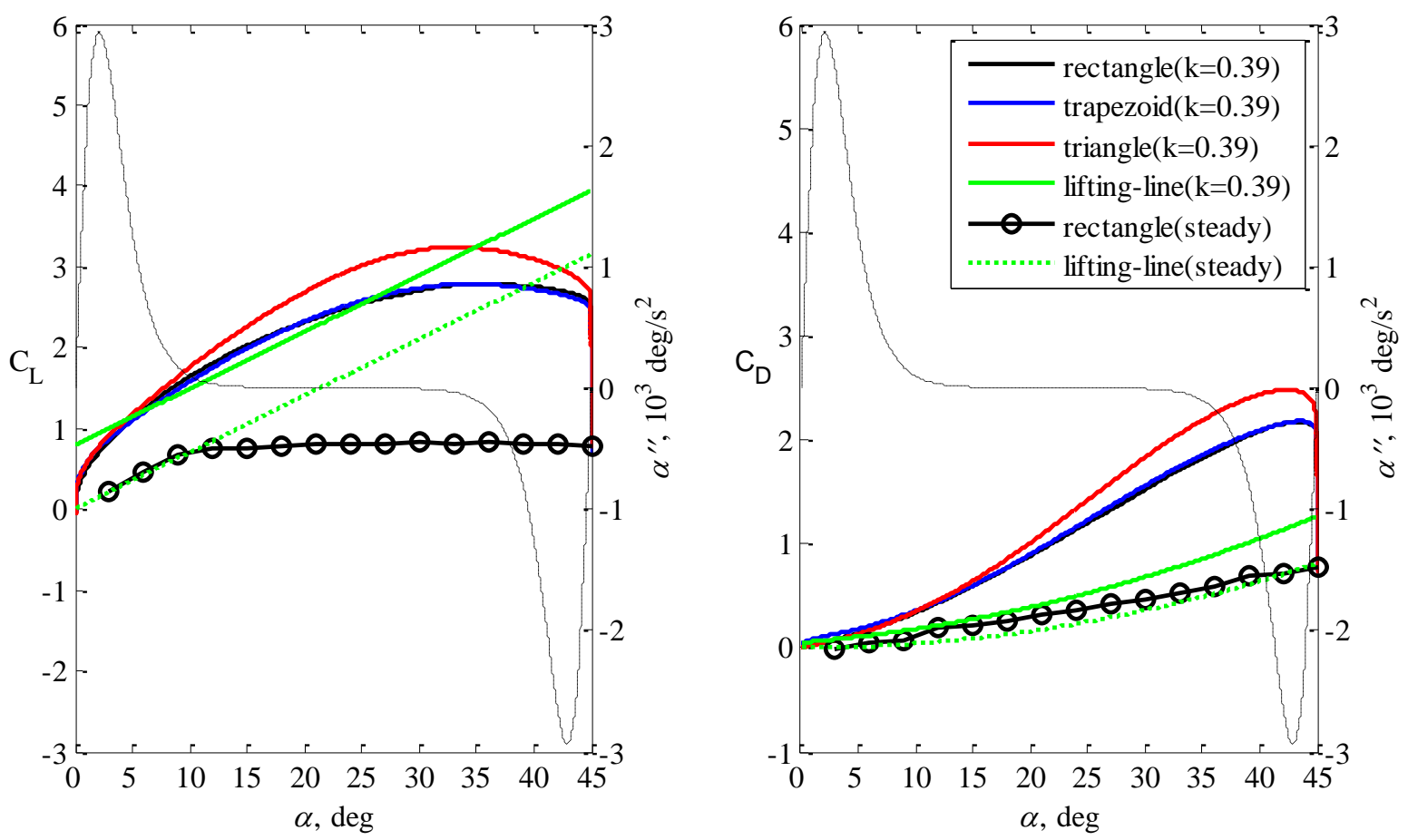

Figure 17 Force evolutions as a function of angle of attack at mid-chord pivot axis for (left) lift coefficient and (right) drag coefficient
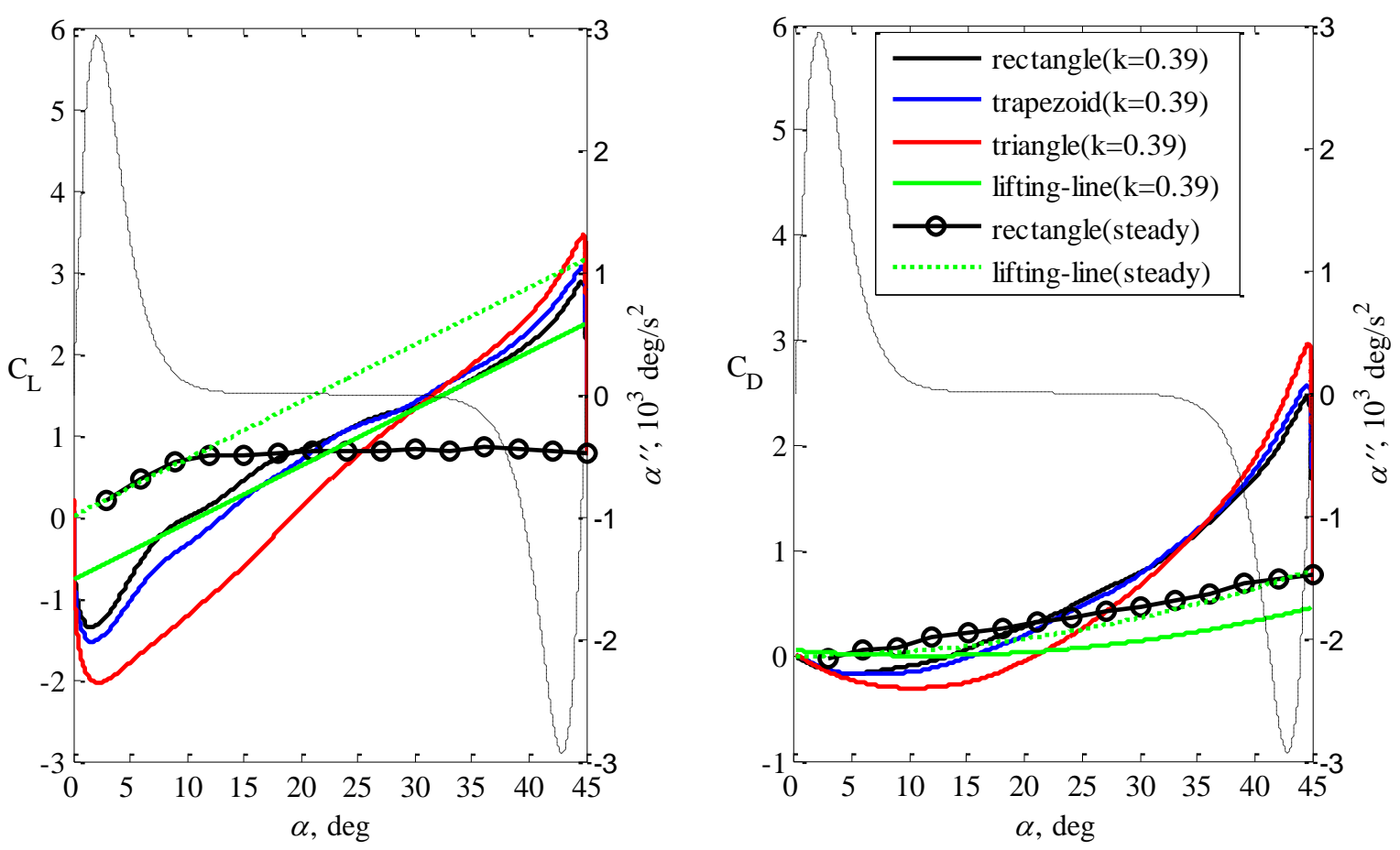

Figure 18 Force evolutions as a function of angle of attack at trailing edge pivot axis for (left) lift coefficient and (right) drag coefficient 

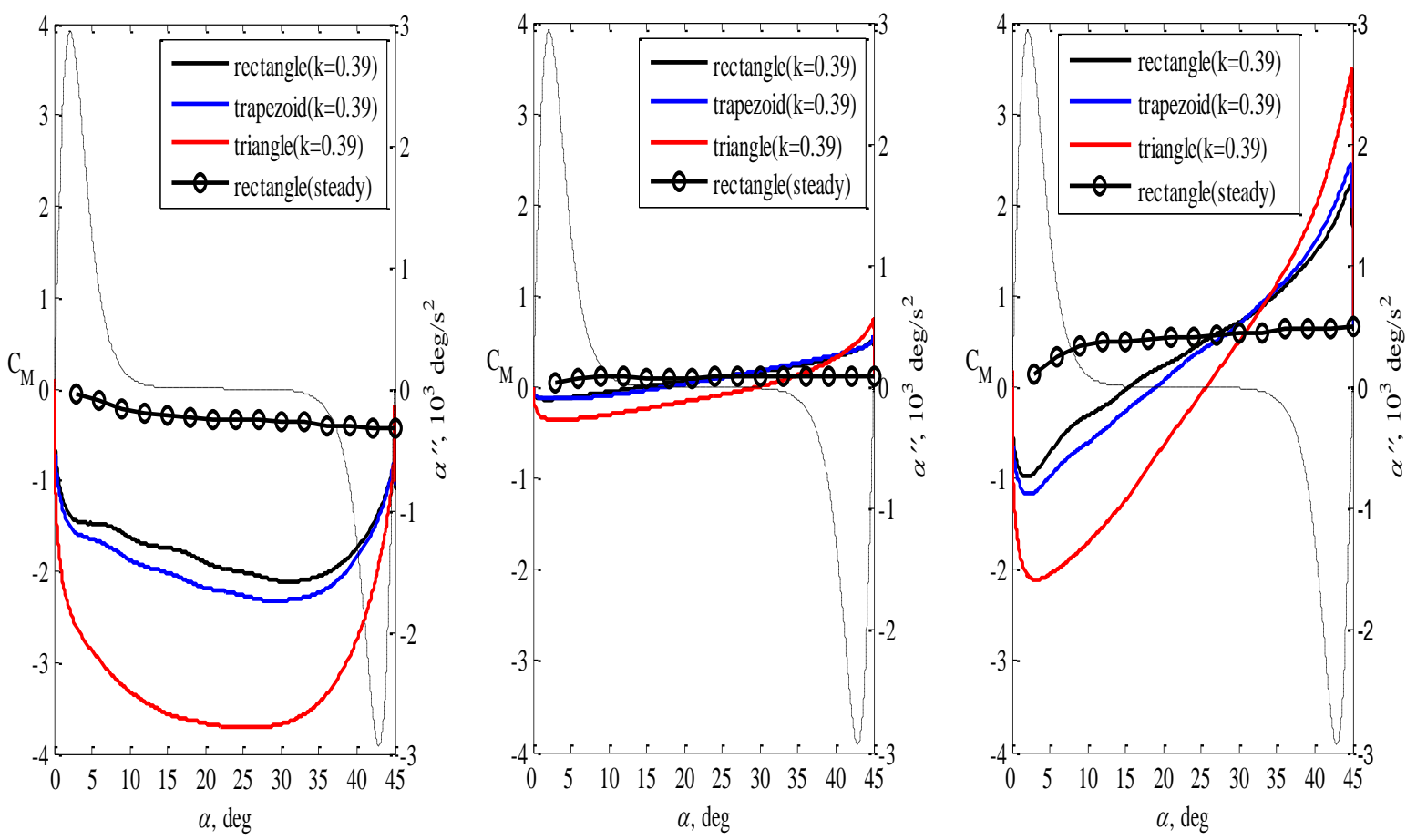

Figure 19 Pitch Moment evolution as a function of angle of attack for pivot axis at (left) leading edge, (middle) mid-chord, and (right) trailing edge.

\section{F. Flow Visualization - Dye Injection}

The flow evolutions over pitching wings are studied using dye injection at $50 \%$ of span with pitch rate $155 \%$ and free-stream velocity $17.5 \mathrm{~cm} / \mathrm{s}(\mathrm{Re}=8.9 \mathrm{k})$, corresponding to reduced pitch rate $K=0.39$. Selected figures are discussed here to illustrate the main flow features between different wing planforms and pivot axis. The injection rake, which is placed at 3 mean chords upstream of leading edge, has 7 probes with 1" spacing, three for red dye and four for blue dye. The center probe releases the red dye to the leading edge of wings at zero angle of attack for all cases considered. Side and top views are used to inspect the flow structure. The side view provides the field of view of the flow about wing chord; the top view gives the field of view of the flow about wing span.

\section{At the end of pitch-up phase $-45^{\circ}$ angle of attack}

Figure 20 shows flow visualization for wings at the end of pitching motion (i.e. at $45^{\circ}$ angle of attack) for leading edge pivot, and all wings have zero leading edge sweep angle.

For the rectangular wing in the side view, there are few vortices in the wake, which formed while the wing was at zero angle of attack. A starting vortex with countclockwise rotation is observed in the wake at a distance about one chord downstream of the trailing edge, which formed at pitch angle of about $11^{\circ}$ and deviated downward from initial chordwise direction. After the formation of starting vortex, the dye from center streakline resides at the rear of wing chord on the suction side of wing surface during pitching motion. Meanwhile, the center streakline near the leading edge deflects upward and swirls over the 3/4 chord of wing, and then become wrinkled before merging with the rest dye. The center streakline behind the merging point moves slightly upward in spanwise direction, as shown in the top view (right panel). The streaklines before the merging process (i.e. lower angle of attack) stay in the same plane, which indicates a two-dimensional flow structure.

For the trapezoidal wing in the side view, there are few vortices in the far wake at zero angle of attack but with different structure compared to the rectangular wing. Unlike the rectangular wing, no dye resides on the suction side of wing surface during the pitching motion, the merging process is not observed. The center streakline over the leading edge of the wing has a similar profile as the rectangular wing. In the top view, these vortices in the far wake move downward, and the center streakline deviates more in spanwise direction compared with rectangular wing. 
For triangular wing in the side view, vortices in the far wake formed before the wing pitched, which is different from the other two wing planforms, more dye diffusion is observed. The center streakline produces a similar structure over the leading edge of wing. In the top view, the center streakline swirls upward along the trailing edge at about one chord downstream of the trailing edge, which indicates stronger axial flow. The streaklines above the center deflects significantly upward from their original path; however, the streaklines below the center go downward. Hence, an oval profile of streaklines forms in the wake, which indicates stronger three dimensional pitch rate effects. The vortices in the far wake stay in the same plane as at early stages.

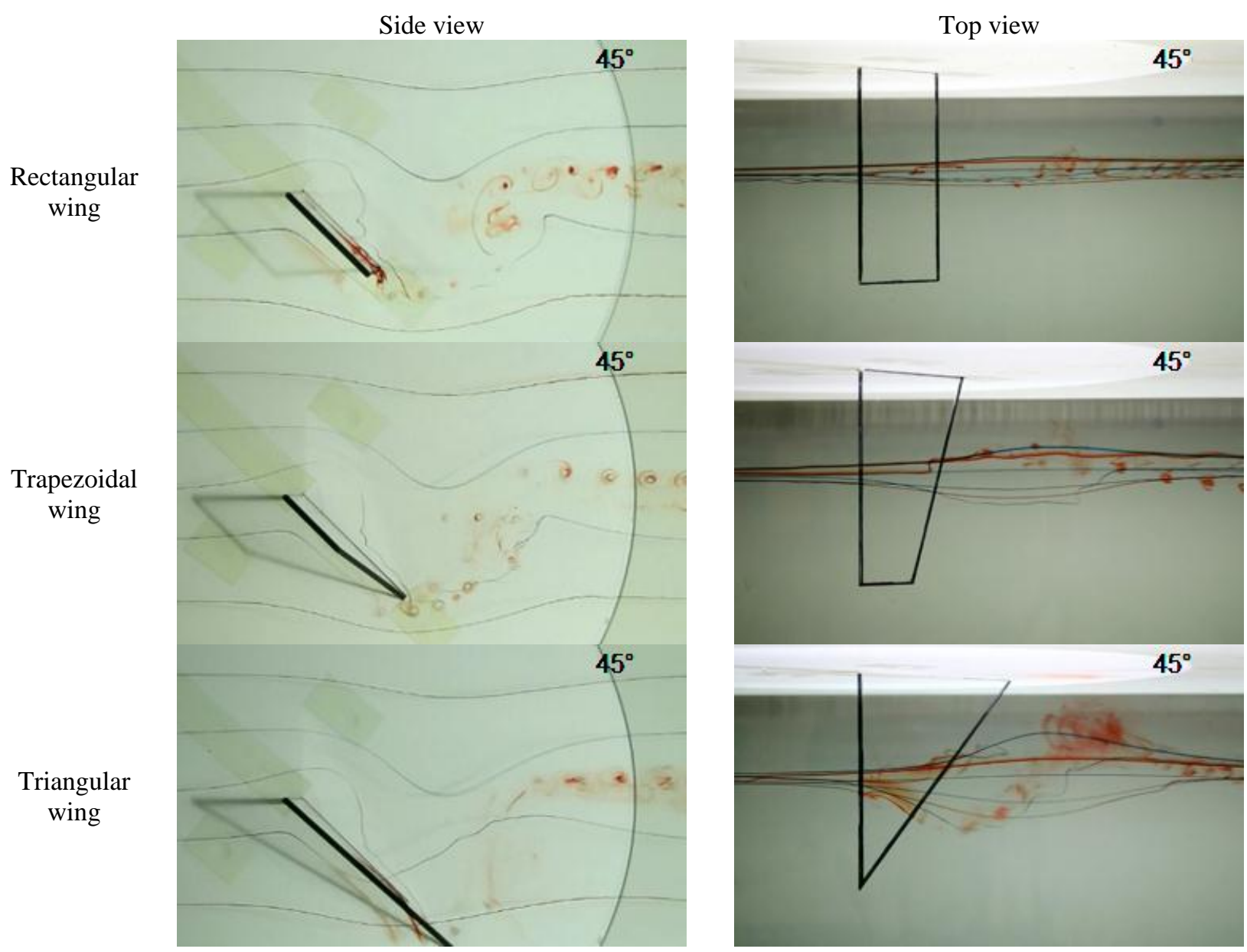

Figure 20 Flow visualization over wings at leading edge pivot and the end of pitch-up phase $45^{\circ}$ angle of attack.

Figure 21 shows flow visualization for wings for trailing edge pivot at $45^{\circ}$ angle of attack. The leading edge sweep angle for rectangular wing, trapezoidal wing, and triangular wing is $0^{\circ}, 18.4^{\circ}$, and $45^{\circ}$, respectively.

For the rectangular wing in the side view, vortices in the far wake were formed at zero angle of attack before the motion started, similar to the leading edge pivot case. A starting vortex is found at about one chord downstream of the trailing edge with clockwise rotation, which is in the opposite direction of the leading edge pivot case, and formed at higher pitch angle of about $21^{\circ}$. This starting vortex in the wake moves upward from the initial chordwise direction, which is contrary to the leading edge pivot case. During the pitching motion some dye from the center streakline is at the rear of wing chord on wing suction side, whereas some dye remains on the pressure side around the quarter chord and forms another starting vortex with counterclockwise rotation close to leading edge. As pitching motion continues, the center streakline upstream moves toward the wing on the pressure side along the chord until $45^{\circ}$ angle of attack is approached. Once this center streakline meets with the dye on the suction side, the formation of the trailing edge vortex with counterclockwise rotation is initiated. The flow evolution during the pitchup phase around the wing is mostly $2 \mathrm{D}$, as shown in the top view. 
For the trapezoidal wing in the side view, the initial flow structure is different compared to the rectangular wing. A starting vortex with counterclockwise rotation is also present in the wake at about one chord downstream of the trailing edge, which was formed at pitch angle $21^{\circ}$ similar to rectangular wing case. During the pitch-up phase some dye from the center streakline remains on the suction side of wing surface; at the same time, the center streakline moves toward the pressure side of the wing and convects along the chord to the trailing edge. Once this center streakline encounters the rest of dye on the suction surface, the formation of a trailing edge vortex is initiated; this evolution is similar to rectangular wing case. There is no indication of a starting vortex at the leading edge in the side view, but there is dye accumulation at the leading edge of the wing surface on pressure side. Also observed on the top view are the streaklines during the pitch-up phase, which stay in the same plane, indicating $2 \mathrm{D}$ flow evolution.

For the triangular wing in the side view, initial vortices in the far wake are different compared to the other two wing planforms. A starting vortex forms in the wake at about one chord downstream of the trailing edge, which is similar to the other wing planforms. No starting vortex at the leading edge is observed from either the side view or the top view. Some dye from the center streakline reside on the suction side of the wing surface during the pitching motion, meanwhile the center streakline moves toward the wing surface on the pressure side and convects to the trailing edge along the wing chord to initiate the formation of a trailing edge vortex. The streaklines from the top view show s 2D flow evolution as in the other two wing planforms.

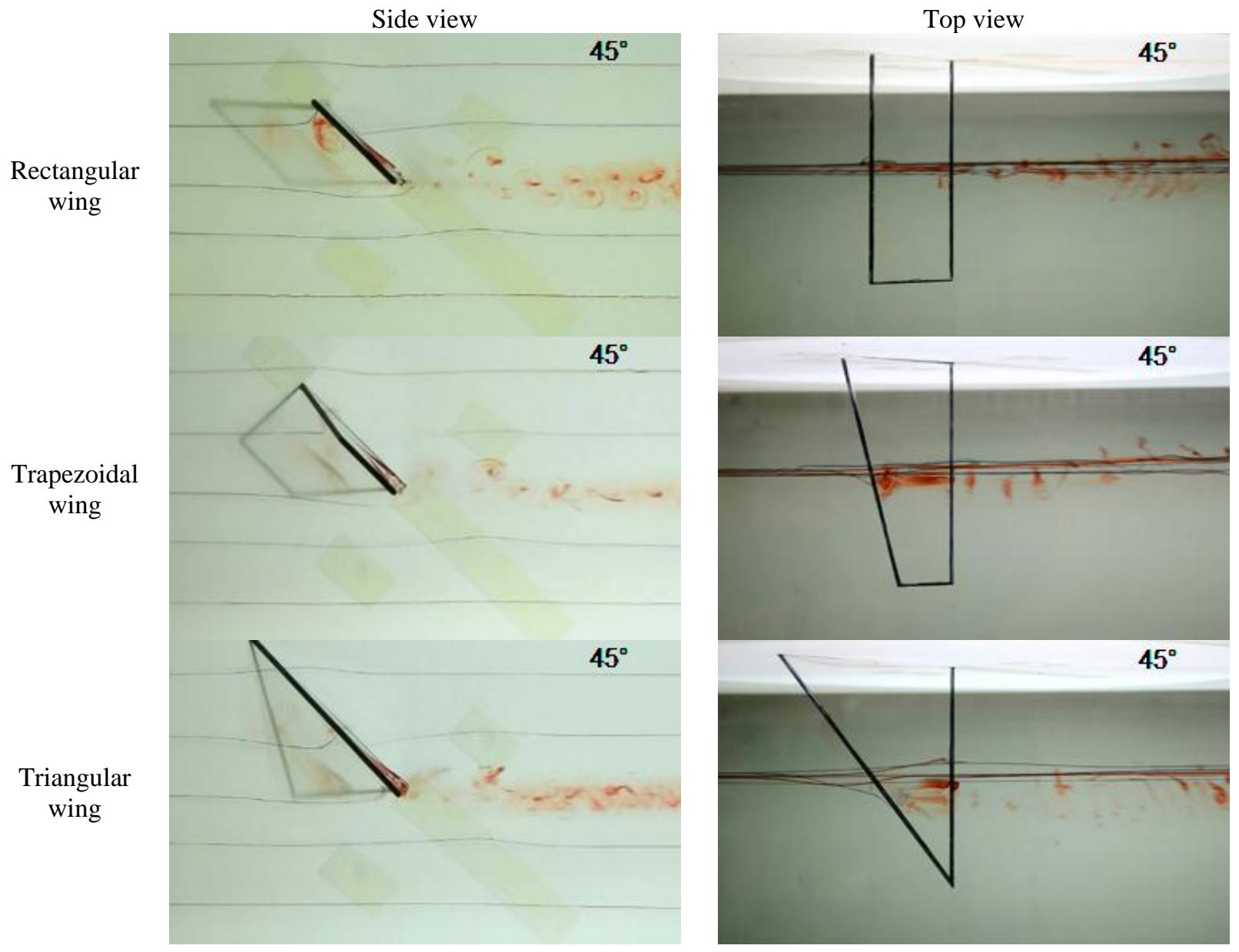

Figure 21 Flow visualization over wings at trailing edge pivot and the end of pitch-up phase $45^{\circ}$ angle of attack.

\section{During the hold phase - 1 convective time after the end of pitch-up phase}

Figures $22-23$ show the flow topology at one convective time after the wing motion stopped at $45^{\circ}$ angle of attack for pivot axis at leading edge and trailing edge, respectively. The corresponding phase is shown by the first vertical line in Fig. $10-12$ before oscillatory force behavior occurs. 
Side view

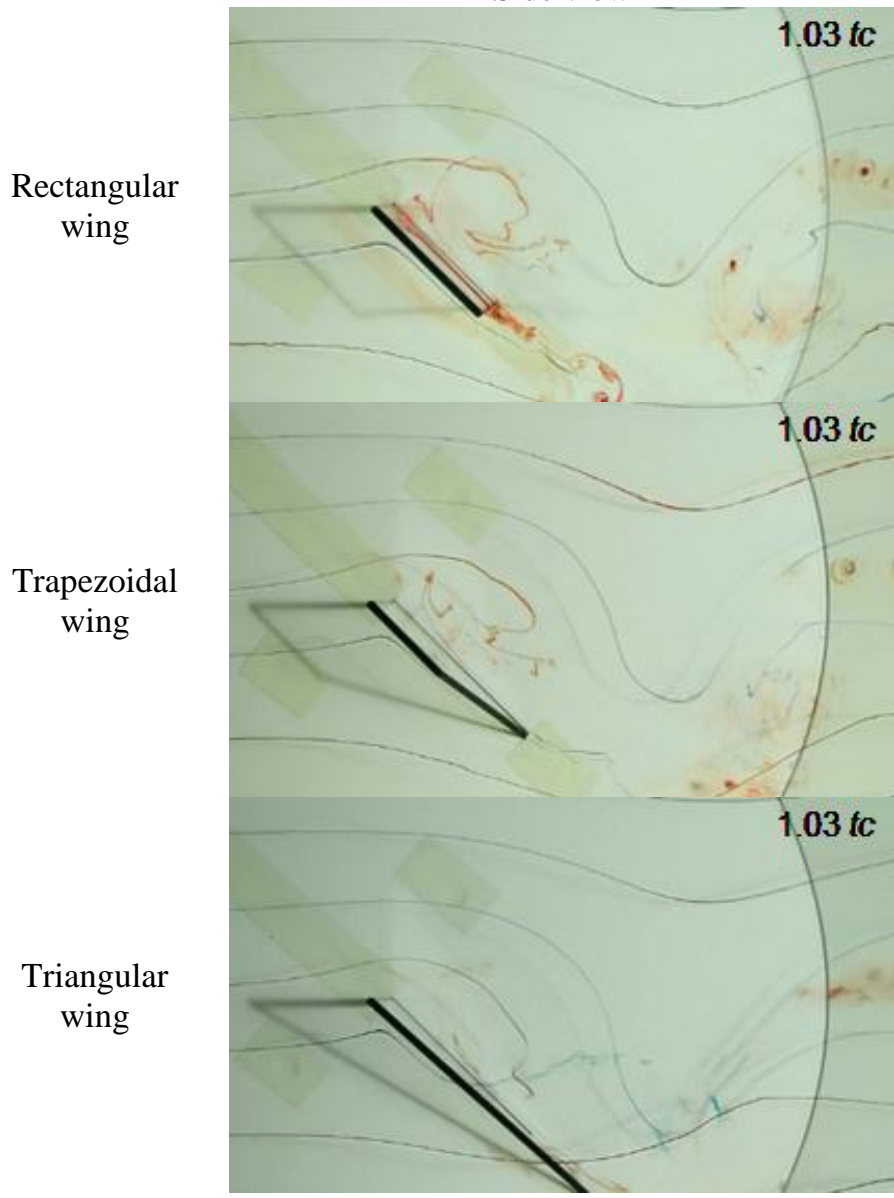

Top view

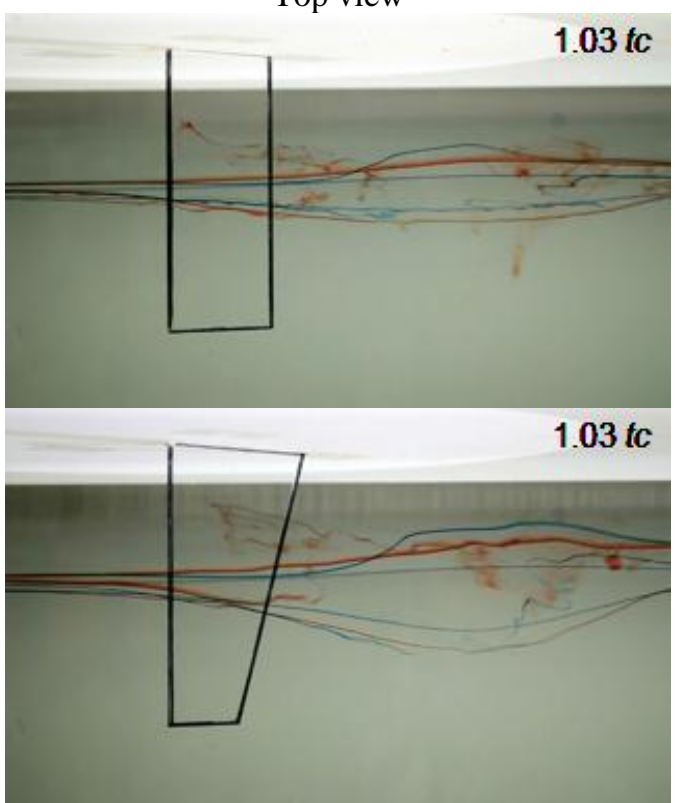

$1.03 \mathrm{tc}$

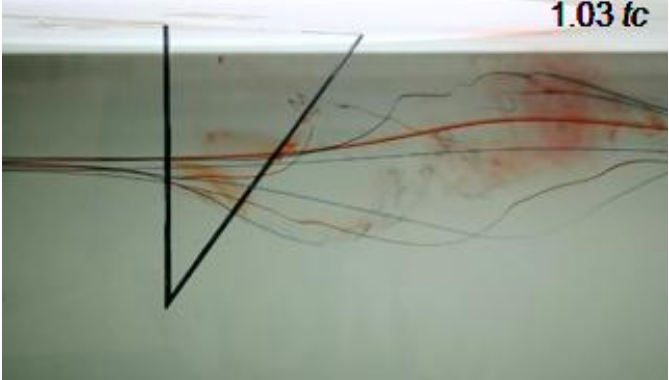

Figure 22 Flow visualization over wings at leading edge pivot during the hold phase: 1 convective time after the end of pitch-up phase.

As shown in Fig. 22, for rectangular wing at leading edge pivot in the side view, the center streakline swirls over leading edge and moves upward in the spanwise direction, which pushes the streaklines on the suction side to curl in the same direction, and the streaklines on pressure side to curl downward. This process displaces the streaklines partially (streamwise direction) in the wake, as shown in the top view. Close to the suction surface, some dye remaining at the rear of wing chord flow reversely toward the quarter chord but still attach to the suction surface, and part of them meet with center streakline upstream at the trailing edge and form a trailing edge vortex, which can be found one chord downstream of the trailing edge in figure after about 1 convective time. The blue streakline below the center streakline moves toward the quarter chord of wing on pressure side of wing surface, and follows the surface downstream. After 3.56 convective times, this blue streakline reveals the formation of another trailing edge vortex, corresponding to the third vertical line in Fig. 10, where normal force is decreased and axial force is increased. In the top view, the starting vortex spins downward and links to wing tip, shown at two chord distance downstream of trailing edge.

For the trapezoidal wing in the side view, the evolution of the center streakline over the leading edge is similar to rectangular wing. At about 1 convective time later, the center streakline swirls upward, and evolves into the direction of normal force at later time; this processing is faster than rectangular wing. There are no dyes resided on wing surface at suction side. The blue streakline below the center streakline, shown in the side view, moves toward the quarter chord on the pressure side of wing surface and follows the surface downstream, which is similar to rectangular wing. The trailing edge vortex is also observed after 3.56 convective times, which corresponds to a decrease of normal force and an increase of axial force. At top view shows the oval profile of steaklines in the wake larger than rectangular wing and its previous times. 
For the triangular wing in the side view, the leading edge swirl covers the wing chord; its evolution is much quicker than the other wing planforms. There is no residual dye on suction side of wing surface. The blue streakline below the center streakline moves toward the quarter chord of wing on pressure side as the other wing planforms and follows the pressure surface downstream. However, unlike rectangular wing and trapezoidal wing, there is no trailing edge vortex observed due to stronger axial flow. The oval profile of streaklines is much larger than the other two wing planforms and less smooth than the profile in previous time.

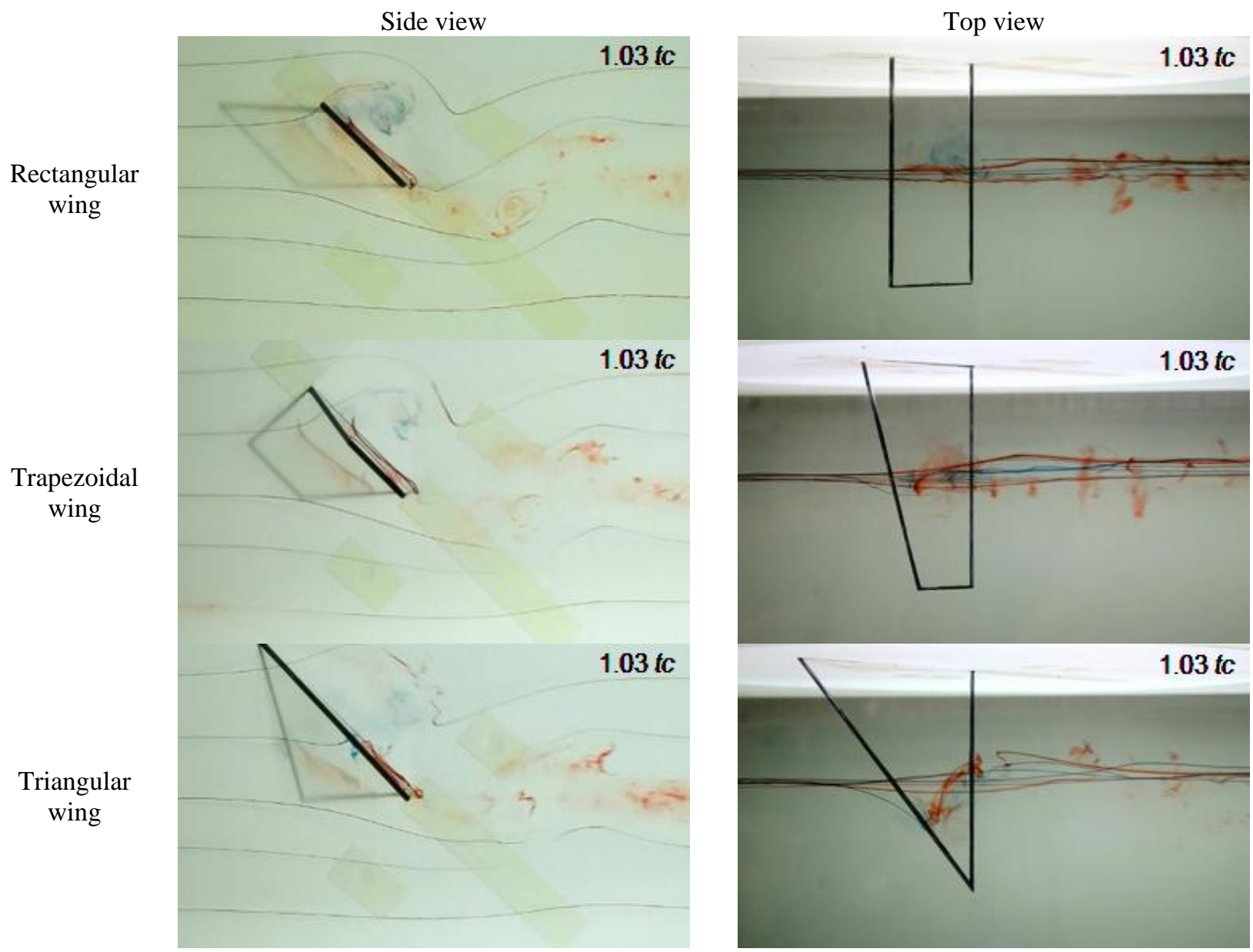

Figure 23 Flow visualization over wings at trailing edge pivot during the hold phase: 1 convective time after the end of pitch-up phase.

Figure 23 shows the flow topology at about one convective time after the wing stop at $45^{\circ}$ for trailing edge pivot. The leading edge sweep angle is $0^{\circ}, 18.4^{\circ}$, and $45^{\circ}$ for rectangular, trapezoidal, and triangular wings, respectively.

For the rectangular wing in the side view, once the center streakline on pressure side of wing meets residual dye on suction side of wing surface at trailing edge, a trailing edge vortex in counterclockwise rotation is going to formed, which is about one chord downstream of trailing edge in figure. The starting vortex at leading edge is stretched into two parts, observed from video, one move to the leading edge on suction side, the other moves downstream along the pressure surface. After about 1 convective time residual dye on wing surface at suction side flows reversely toward the quarter chord of wing and forms the secondary vortex. This dye diffuses eventually at quarter chord along the span. In addition, the blue streakline above the center streakline swirls over the leading edge, mixing with red dye from the starting vortex at leading edge. This leading edge swirl curls upward along the span at later time and diminishes as steady state is approached; this evolution is similar to leading edge pivot. Trailing edge swirl by trailing edge vortex is not captured by present dye injection configuration.

For the trapezoidal wing in the side view, a trailing edge vortex is formed when the center streakline meets with the rest of dye on the suction side of wing surface, which is one chord downstream of trailing edge in figure. The starting vortex at leading edge, being found using rectangular wing, is not observed. The dye accumulation formed 
near leading edge during the pitch-up phase on pressure side of wing surface as shown in Fig. 20 convected and diffused downstream. After 1 convective time, the dye at the rear of wing chord on the suction side of wing surface flows reversely toward the quarter chord and diffuse along leading edge in spanwise direction, which is similar to the rectangular wing.

For the triangular wing in the side view, similar to the rectangular wing and trapezoidal wing, the trailing edge vortex is formed as the center streakline encounters the rest of dye at the rear of wing chord on suction side of wing surface. The remaining dye on suction side of wing surface flows toward leading edge and encounter blue streakline from the upstream at a location half of chord from wing tip after about 1 convective time. The formation of leading edge swirl is hard to identify from the blue streakline above the center streakline as the other wing planforms. At later time, this red dye is diffused toward wing tip and convected downstream.

\section{During the hold phase - 27 convective time after the end of pitch-up phase}

From force measurements shown in Fig. 10 - 12, the steady normal force and axial force are achieved after 27 convective times as shown as the last vertical line, where pivot axis effects are absent and tapper ratio effects are small. The corresponding flow topologies are given here for leading edge pivot and trailing edge pivot in Fig. 24 and Fig. 25, respectively.

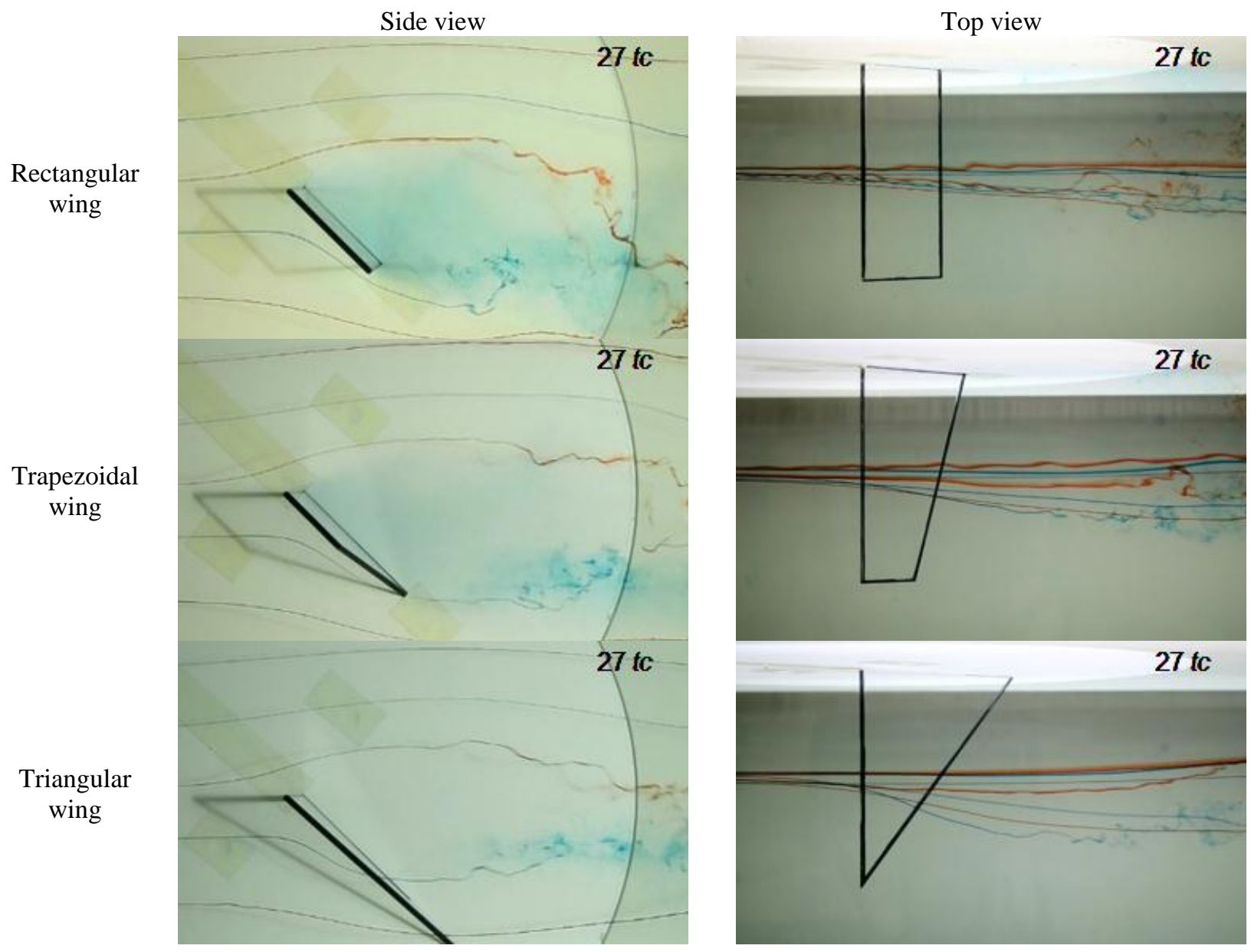

Figure 24 Flow visualization during the hold phase with wings at leading edge pivot: 27 convective times after the end of pitch-up phase.

For the rectangular wing in the side view shown in Fig. 24, the leading edge swirling, residual dye at the rear of wing chord on wing surface at suction side, the formation of a trailing edge vortex, and the oval structure of streaklines in the wake are not observed, even for trapezoidal and triangular wings. The center streakline and blue streakline below the center streakline go around the wing, which stay almost in the same plane as shown in top view. For the trapezoidal wing in the side view, the streaklines around the wing have similar profile with less dye diffusion 
on suction side of wing compared to the rectangular wing case; however, the streaklines at lower position in the side view flow downward across the wing downstream. For the triangular wing in the side view, the streaklines flow over the wing with closer distance apart, which is because the streaklines at lower position in the side view deflect downward much more.

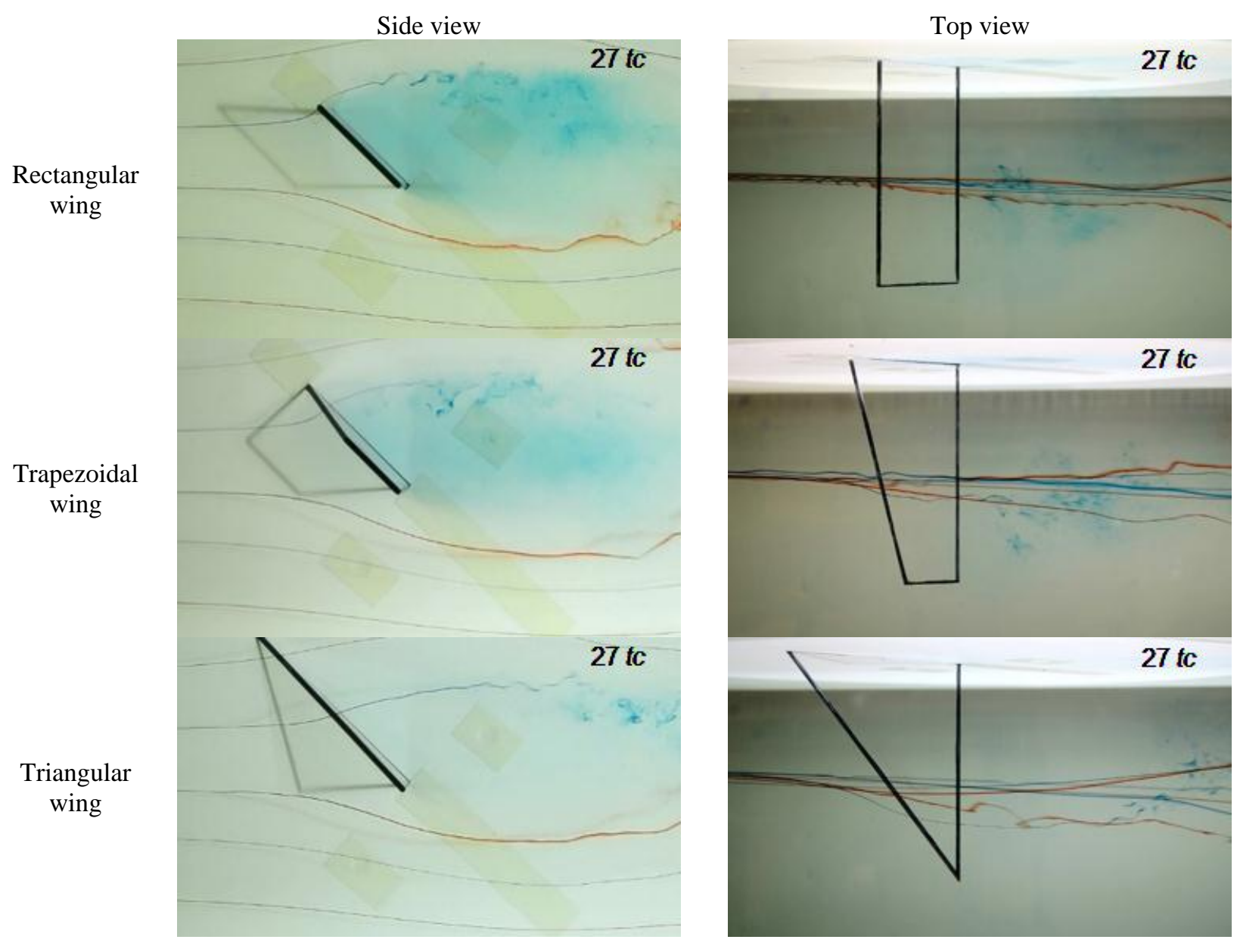

Figure 25 Flow visualization during the hold phase with wings at trailing edge pivot: 27 convective times after the end of pitch-up phase.

For the rectangular wing in the side view shown in Fig. 25, the unsteady flow topology such as leading edge swirling, residual dye on the suction side of wing surface, the formation of the starting vortex at leading edge, and the formation of a trailing edge vortex are not observed. The center streakline and blue streakline above the center streakline are far apart across the wing in the wake; they are closer for the rectangular and the triangular wings because steaklines deflect toward wing tip, less dye diffusion is also observed as tapper ratio decreases.

\section{Conclusion}

We have conducted direct force measurements and flow visualization experiments for finite aspect ratio wings with effective aspect ratio 4 and reduced pitch rate $K=0.39$. Three wing planforms are considered: rectangular, trapezoidal, and triangular. The conclusions of this study are summarized in the following.

From the aerodynamic force measurements,

1. for steady flow measurements (i.e. $K=0$ ),

a. For tapper ratio higher than 0.5 , the lift coefficient follows the lifting-line theory up to $9^{\circ}$. Lower tapper ratio yields lower lift coefficient. 
b. For trailing edge pivot, tapper ratio higher than 0.5 gives stall angle higher than $15^{\circ}$.

c. Drag coefficients are mostly independent of leading-edge-sweep angle and tapper ratio, and increase linearly with angle of attack.

d. Pitch moment coefficient about pivot axis is negative for leading edge pivot, and positive for mid chord and trailing edge pivot. Effects of tapper ratio are small at lower angle of attack.

2. for unsteady flow measurements (i.e. $K=0.39$ ),

a. Normal force increases rapidly during the pitch-up motion and decrease to steady state more gradually after approximate 30 convective times, which is consistent with our previous work in Ref. [18].

b. Transient oscillatory normal force and axial force are measured for the higher tapper ratio wing after the wing reaches the maximum pitch angle and before steady state is reached, possibly due to incipient vortex shedding Ref. [18].

c. Lift and drag coefficients during the constant pitch rate part of the motion show strong dependence on wing planform geometry and pivot axis location. Pivot axis effects are consistent with prior work Ref. [18]. For fix pivot axis force coefficients increase as taper ratio decreases.

d. For a given pivot axis, all wing planforms have similar tendency in force development during the pitch-up phase. For leading edge pivot, the triangular wing (taper ratio 0) produces much higher lift and drag than the trapezoidal wing and rectangular wing, which is well beyond estimates based on lifting-line theory. For mid-chord pivot and taper ratio higher than 0.5 give the same force coefficients, which do not follow the theoretical result.

e. Large negative pitching moments about the pivot axis are measured for leading edge pivot axis. As for the other aerodynamic forces the negative pitching moment increases as taper ratio decreases.

f. For mid-chord pivot axis the pitching moment about the pivot axis is negative for small pitch angle and positive for large pitch angle. The magnitude is larger for the triangular wing compared to the rectangular and trapezoidal wing.

From flow visualization at 50\% wing span,

1. During the pitch-up phase,

a. The flow is substantially $2 \mathrm{D}$.

b. The strength of the starting vortex at the trailing edge of the wing depends on the taper ratio. Higher taper ratio produces stronger starting vortex formation.

c. For leading edge pivot, the initial starting vortex forms in the wake near the trailing edge at pitch angle $11^{\circ}$ with counterclockwise rotation. Taper ratio does not change formation of the leading edge vortex. This result is consistent with PIV measurement, Ref [18].

d. For trailing edge pivot axis, a starting vortex forms in the wake near the trailing edge at pitch angle $21^{\circ}$ with clockwise rotation. A vortex forms at about the quarter chord on the pressure side of the wings as documented by PIV measurements in Ref [18].

2. During the pitch-hold phase,

a. The evolution of the starting vortex at the trailing edge differs depending on taper ratio.

b. The flow visualization results suggest that the trailing edge vortex is linked to the tip vortex for the rectangular wing. For other cases the development of streamwise swirl is more pronounced.

c. Flow visualization suggests that the three-dimensional flow development is enhanced as the taper ratio is decreased. 


\section{Acknowledgments}

The authors would like to give thanks to Dr. Kenneth Granlund from the Air Force Research Laboratory (AFRL) for many fruitful discussions of the research reported in this paper. The work was sponsored in part by the Air Force Office of Scientific Research's Multidisciplinary University Research Initiative (MURI), contract number FA955007-1-0547 and by the Michigan/AFRL Collaborative Center in Aeronautical Sciences. 


\section{Appendix A Flow Visualization during Pitch-Up Phase}

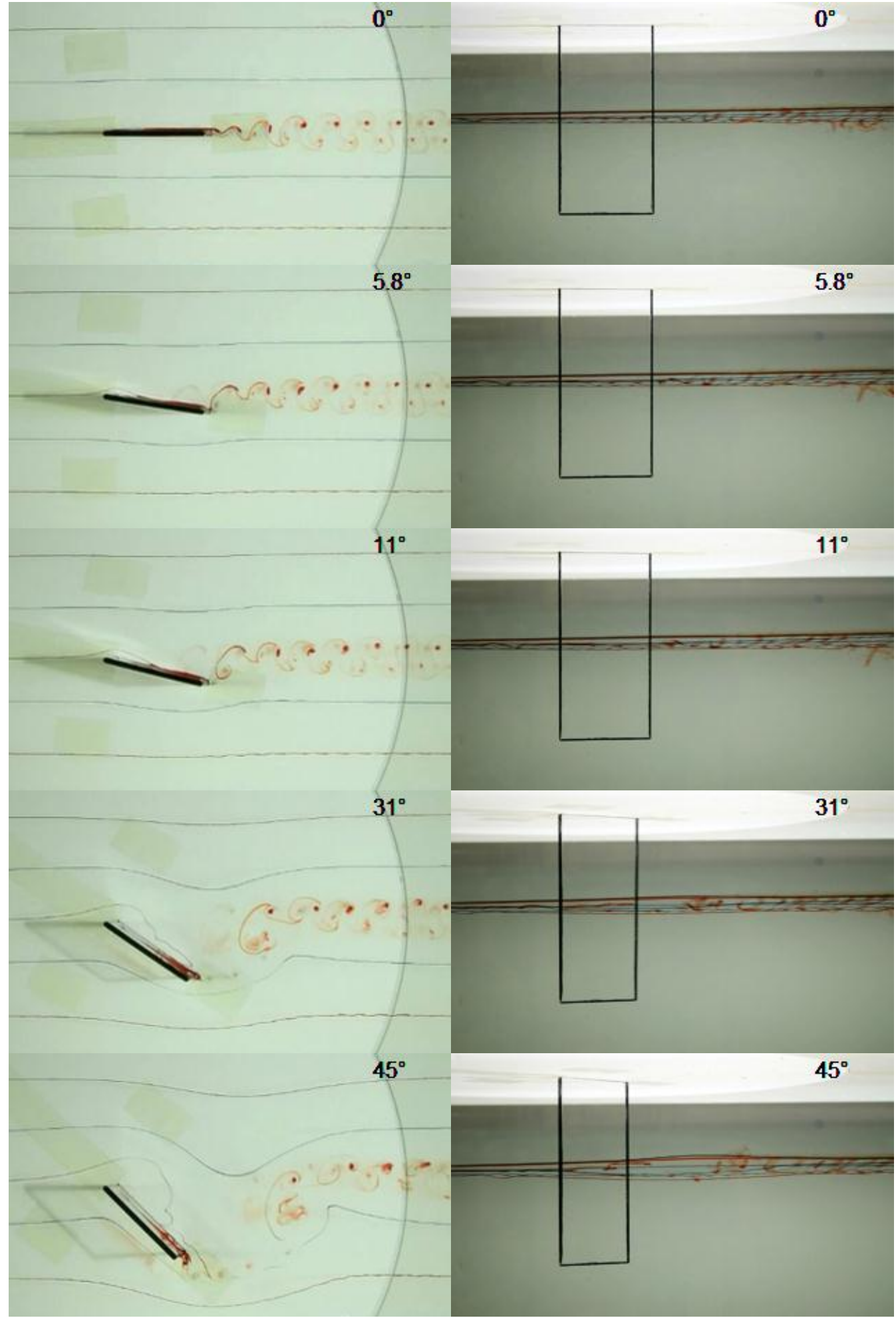

Figure A.1 Flow evolutions during pitch-up at selected phase for rectangular wing at leading edge pivot 


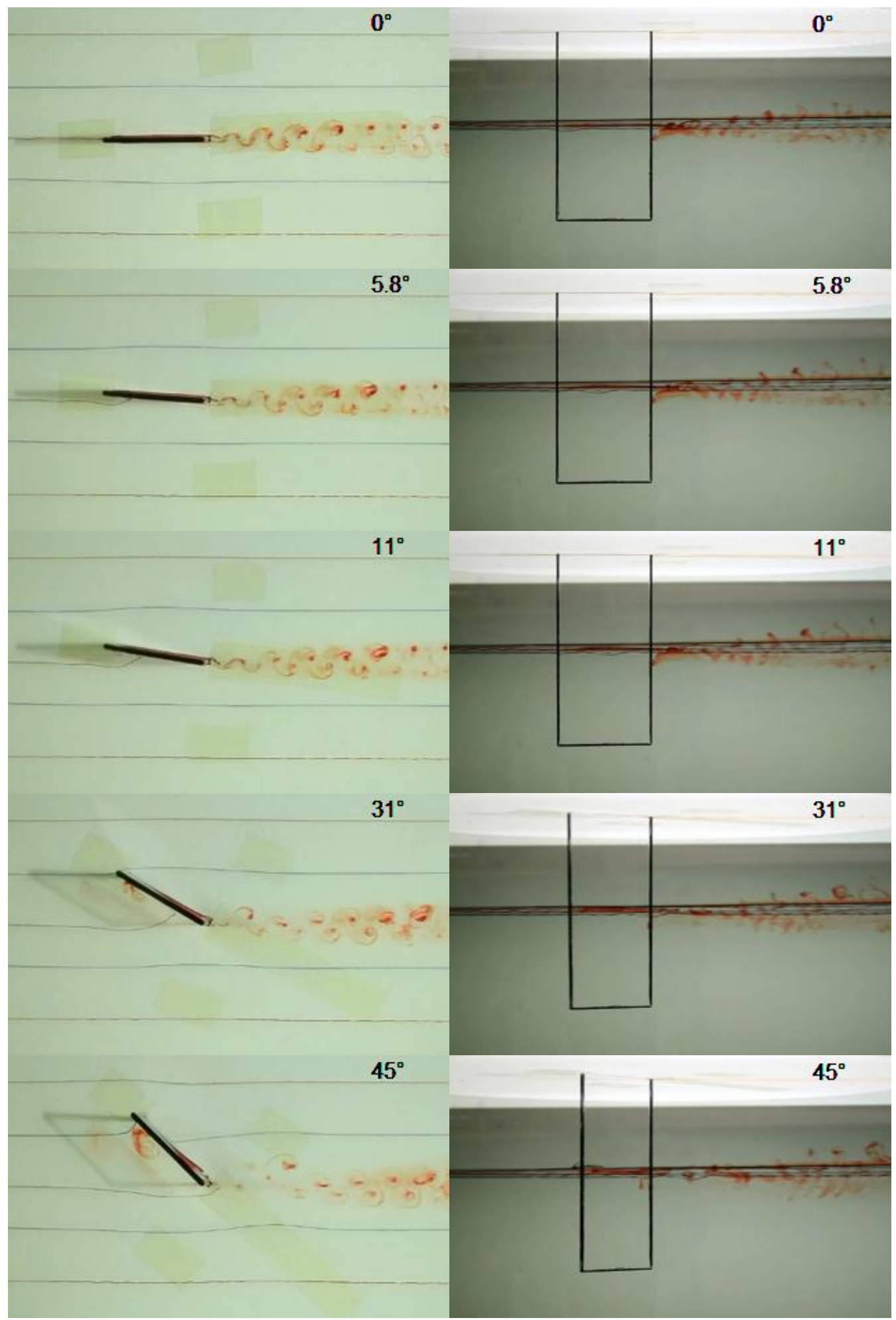

Figure A.2 Flow evolutions during pitch-up at selected phase for rectangular wing at trailing edge pivot 


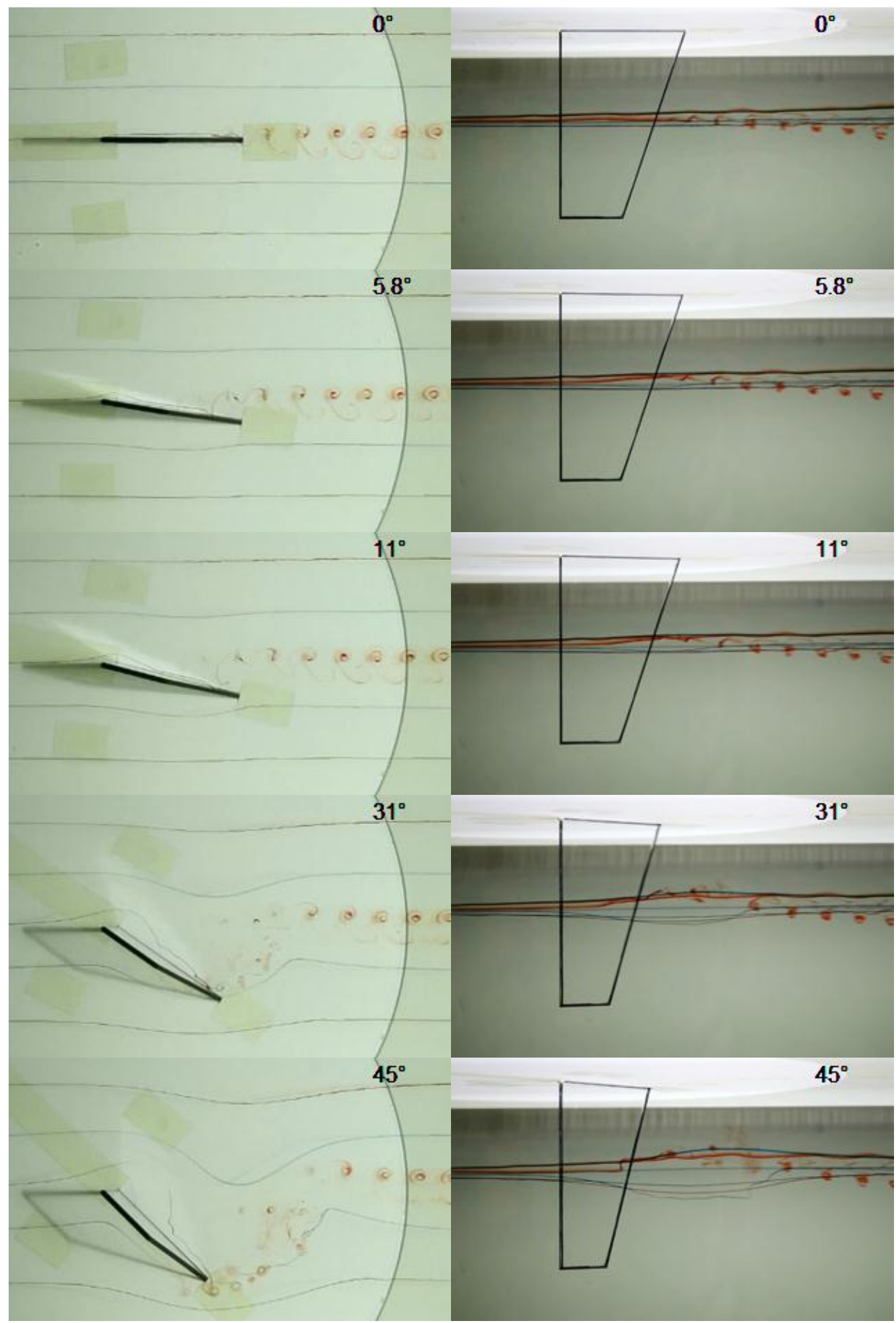

Figure A.3 Flow evolutions during pitch-up at selected phase for trapezoidal wing at leading edge pivot 


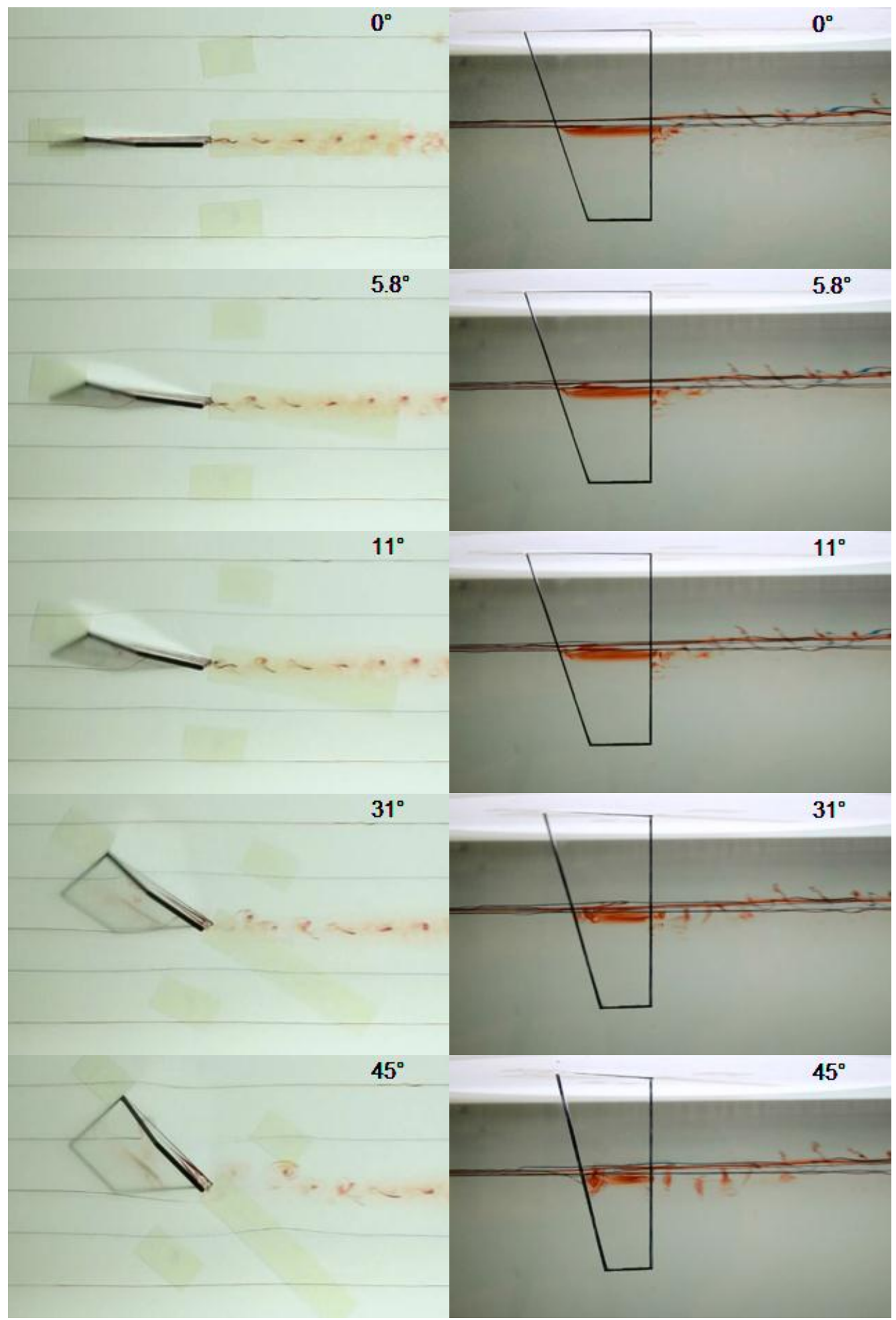

Figure A.4 Flow evolutions during pitch-up at selected phase for trapezoidal wing at trailing edge pivot 


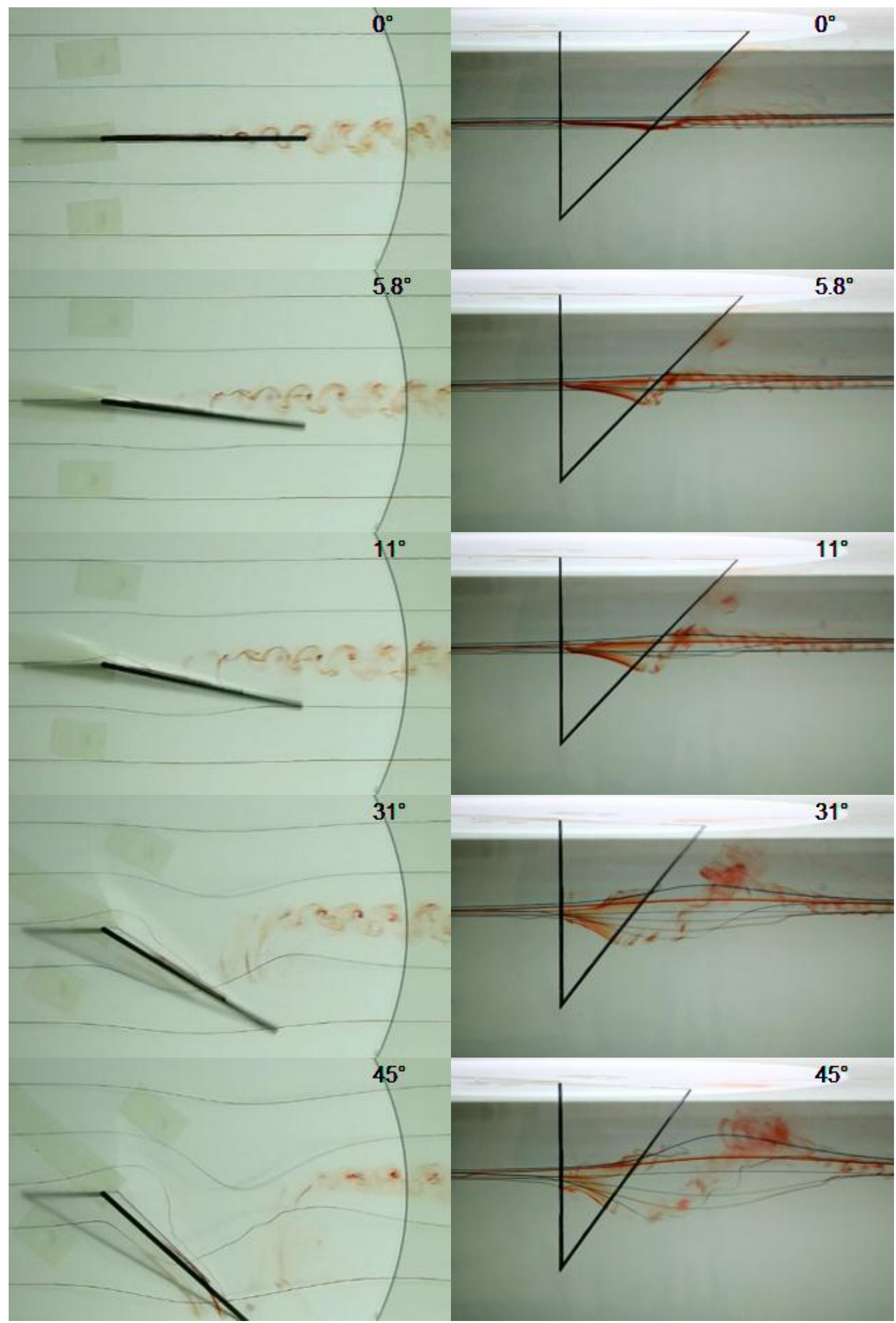

Figure A.5 Flow evolutions during pitch-up at selected phase for triangular wing at leading edge pivot 


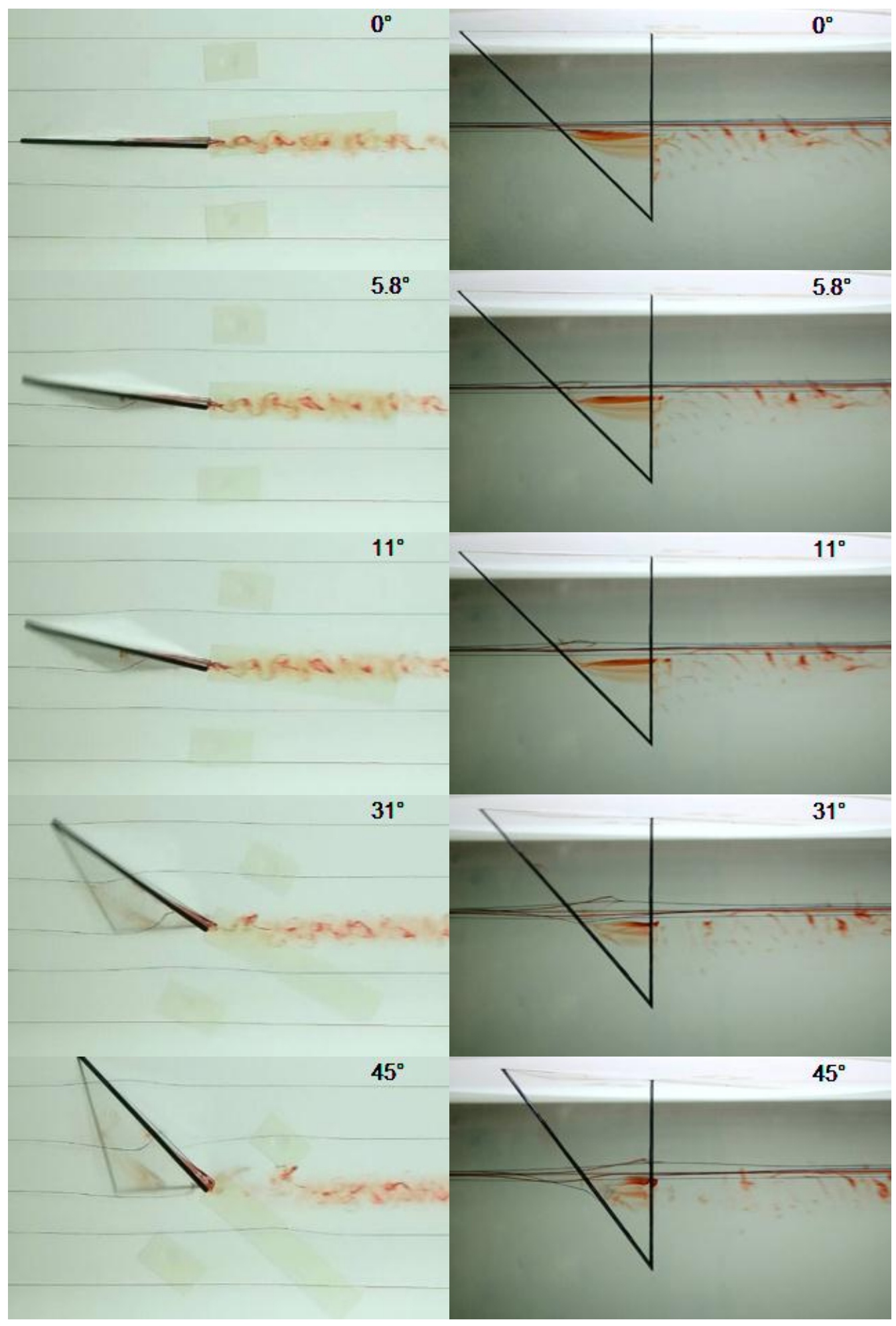

Figure A.6 Flow evolutions during pitch-up at selected phase for triangular wing at trailing edge pivot 


\section{Appendix B Flow Visualization during Pitch-Hold Phase}

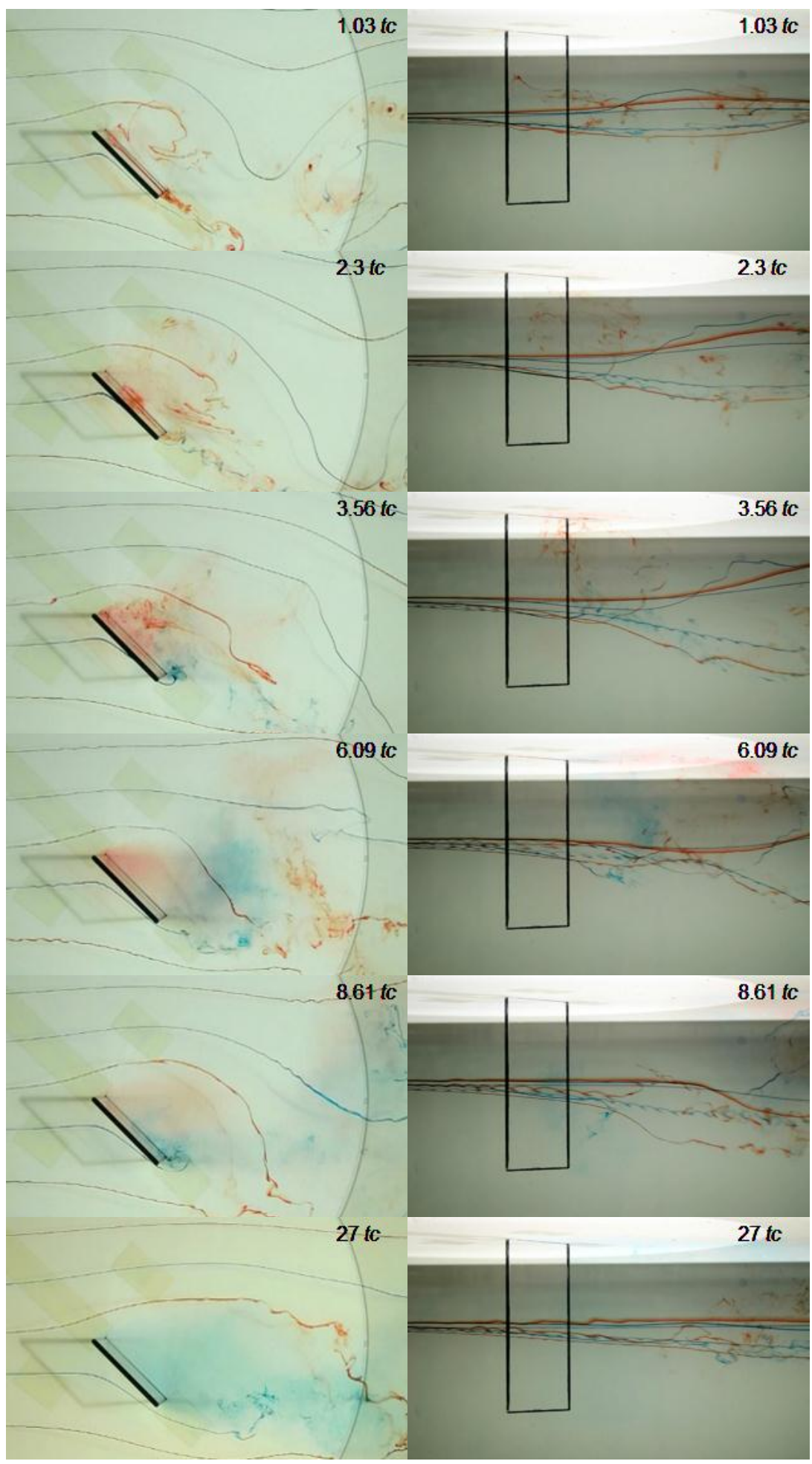

Figure B.1 Flow evolutions at selected phase after motion stop for rectangular wing at leading edge pivot. The timing starts when motion stops at $45^{\circ}$. 


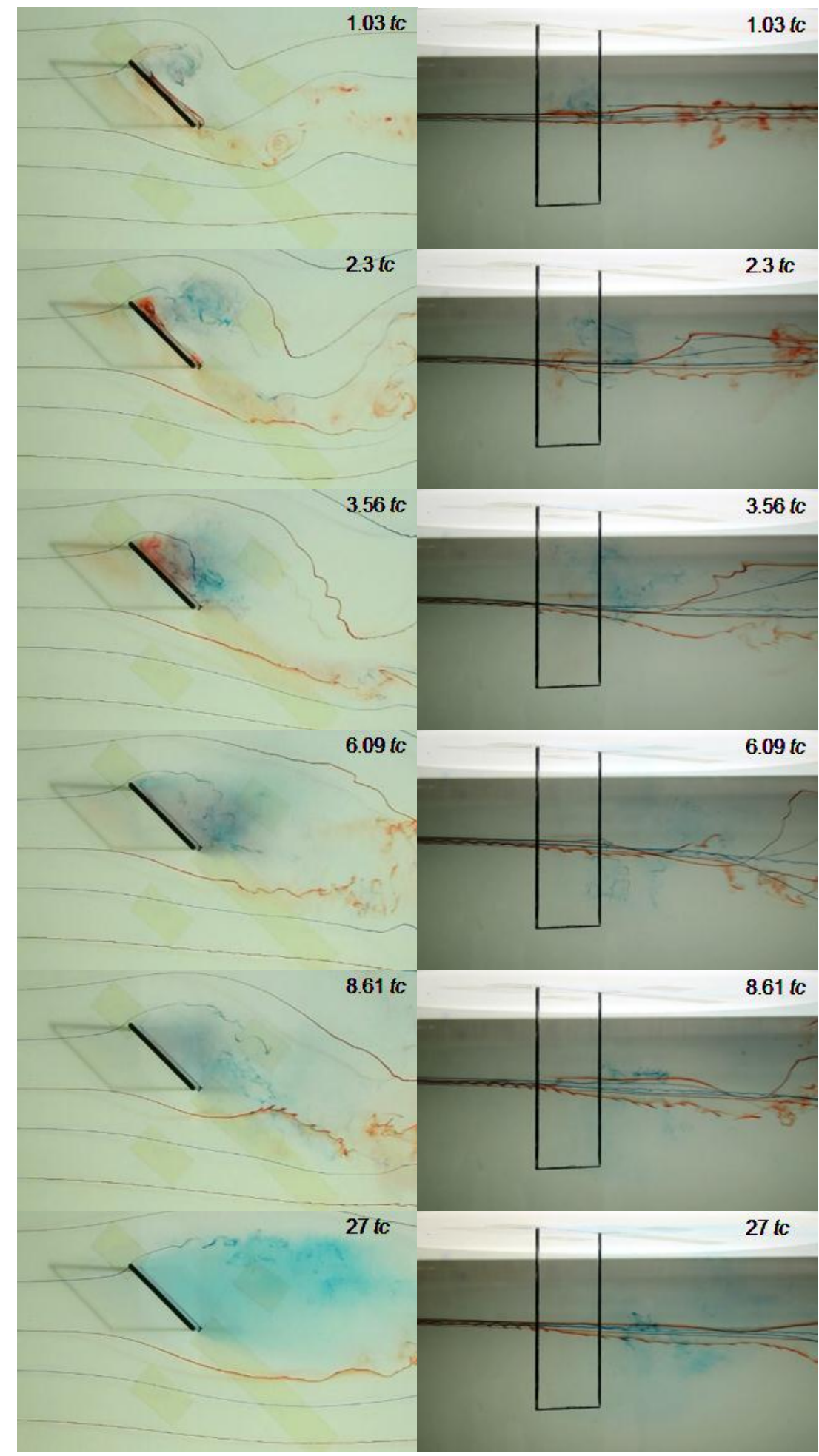

Figure B.2 Flow evolutions at selected phase after motion stop for rectangular wing at trailing edge pivot. The timing starts when motion stops at $45^{\circ}$. 


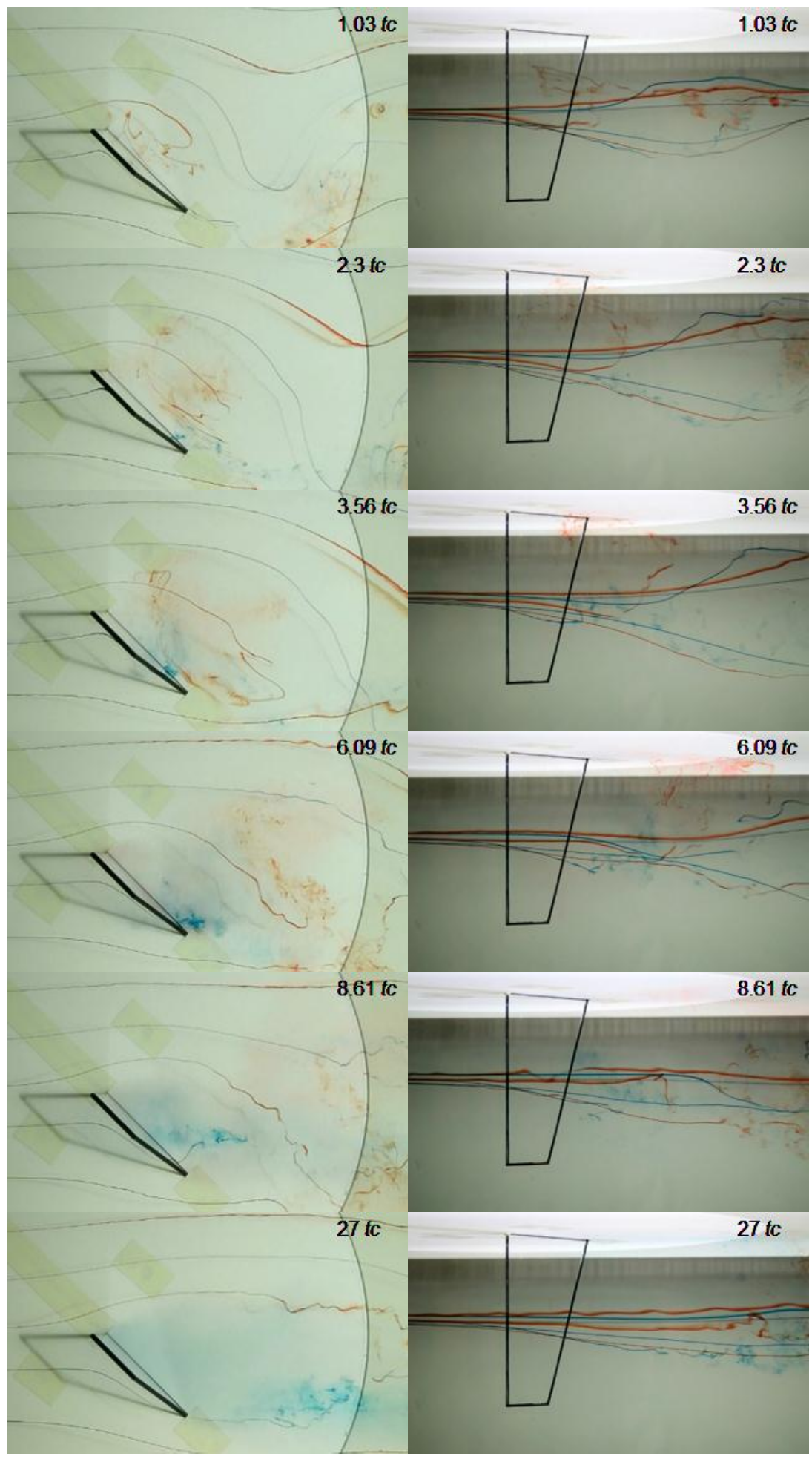

Figure B.3 Flow evolutions at selected phase after motion stop for trapezoidal wing at leading edge pivot. The timing starts when motion stops at $45^{\circ}$. 


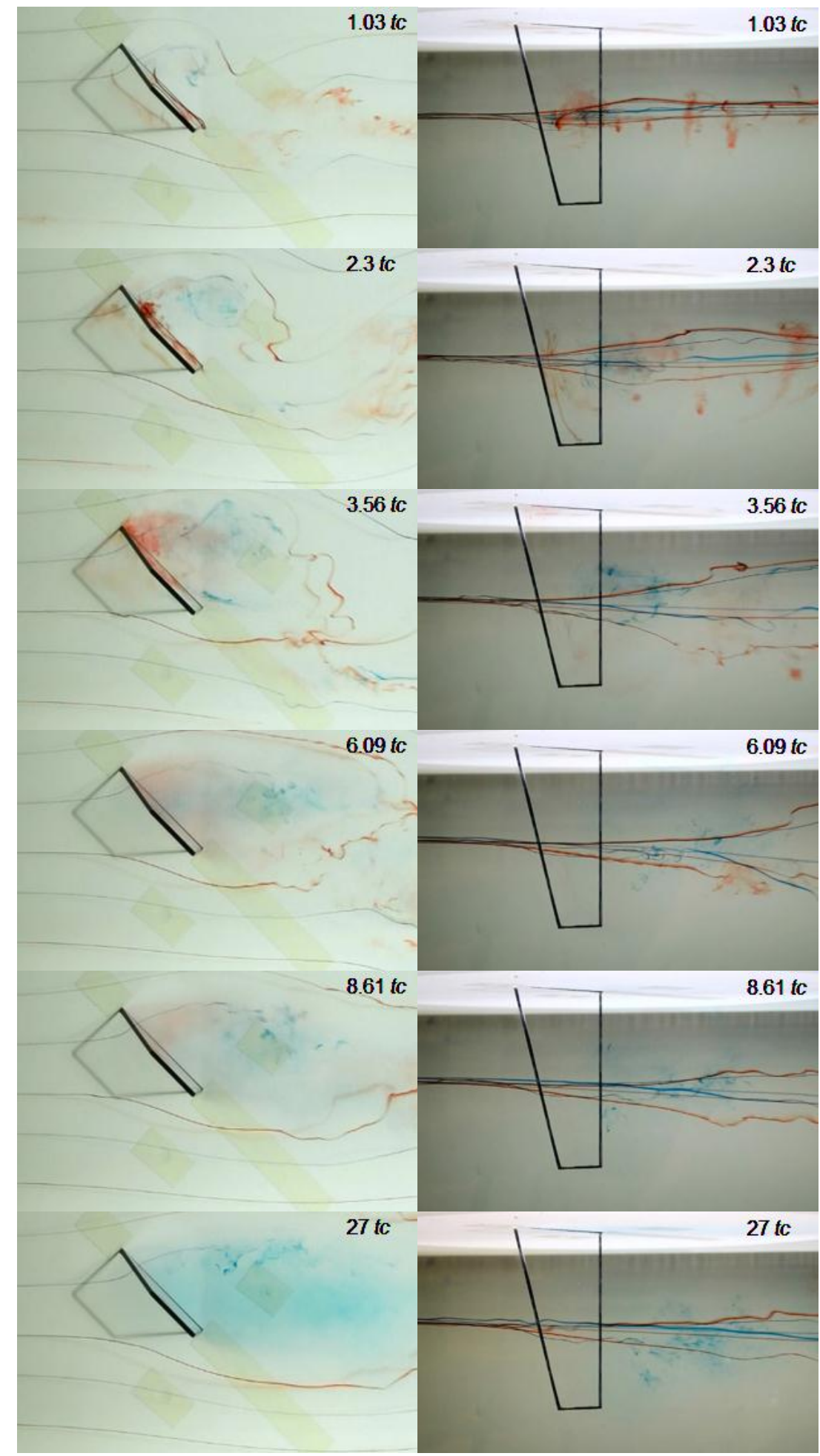

Figure B.4 Flow evolutions at selected phase after motion stop for trapezoidal wing at trailing edge pivot. The timing starts when motion stops at $45^{\circ}$. 


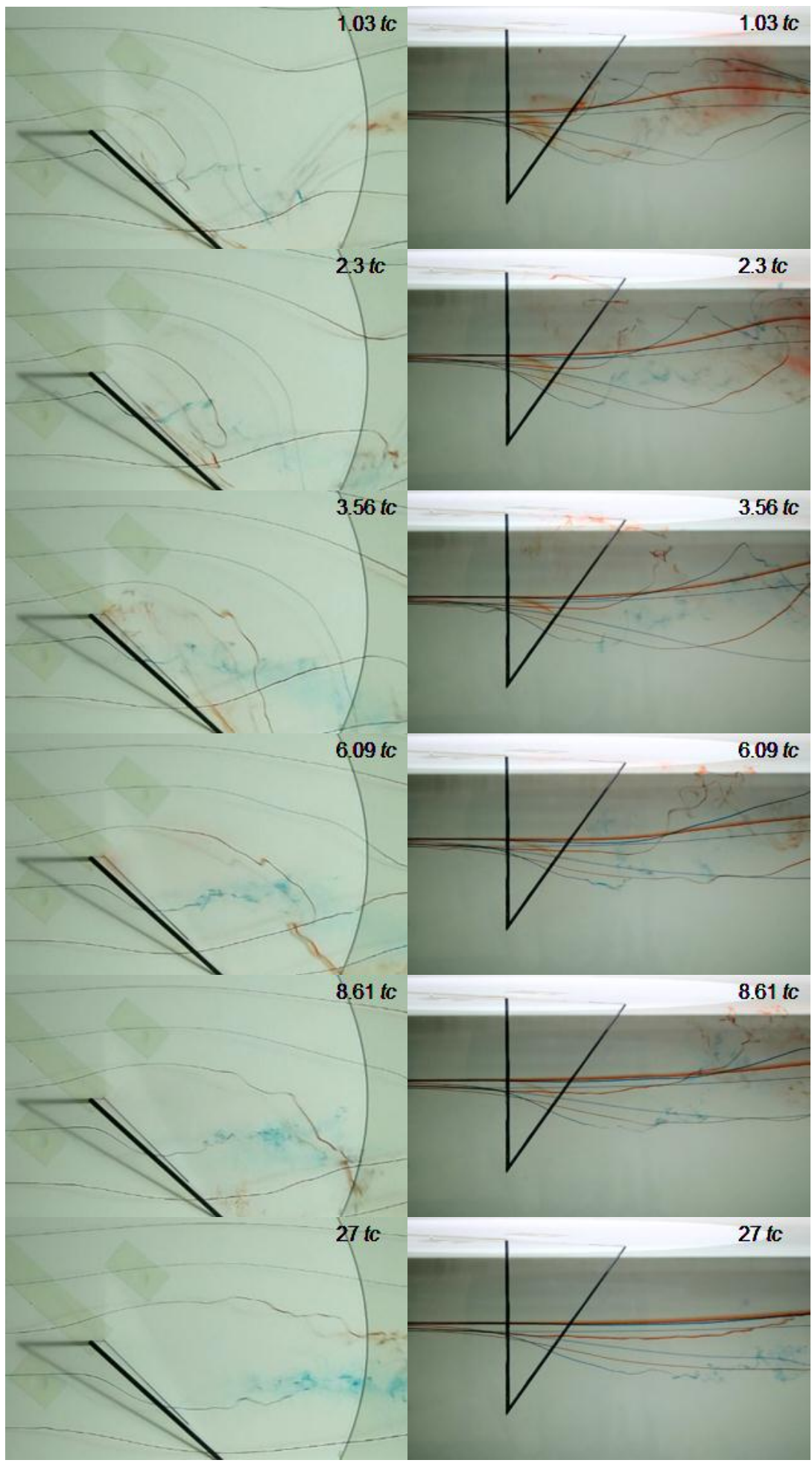

Figure B.5 Flow evolutions at selected phase after motion stop for triangular wing at leading edge pivot. The timing starts when motion stops at $45^{\circ}$. 


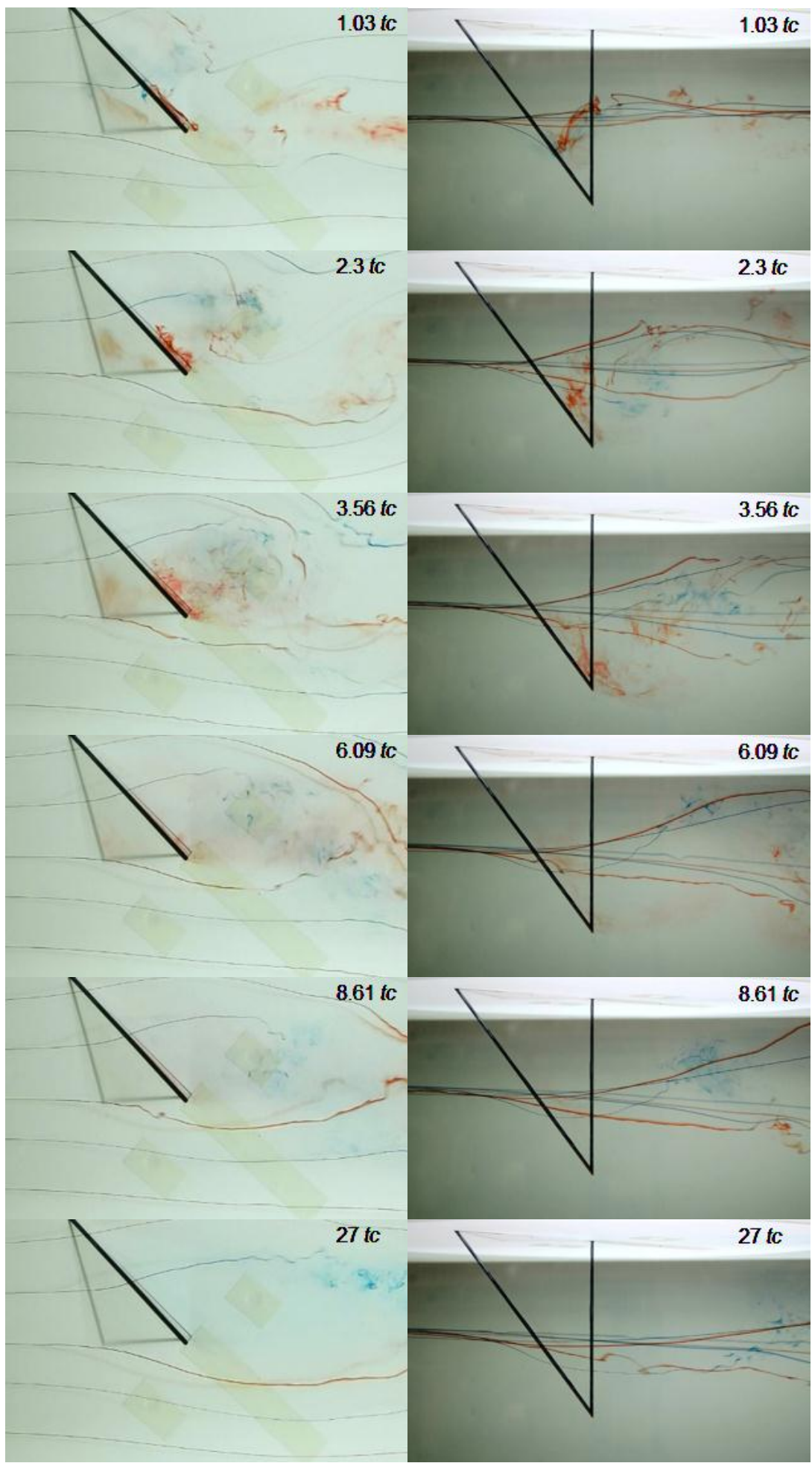

Figure B.6 Flow evolutions at selected phase after motion stop for triangular wing at trailing edge pivot. The timing starts when motion stops at $45^{\circ}$. 


\section{References}

${ }^{1 .}$ Strickland, J.H. and Graham, G.M., "Force Coefficients for a NACA-0015 Airfoil Undergoing Constant Pitch Rate Motions", AIAA Journal, Vol. 25, No. 4, 1987, pp. 662-624.

2. Graham, G.M. and Strickland, J.H., "An Experimental Investigation of an Airfoil Pitching at Moderate to High Rates to Large Angles of Attack", 24th AIAA Aerospace Science Meeting, AIAA-86-0008.

${ }^{3}$.Strickland, J.H. and Graham, G.M., "Dynamic Stall Inception Correlation for Airfoils Undergoing Constant Pitch Rate Motions", AIAA Journal, Vol.24, No.4, 1985, pp. 678.

${ }^{4}$.Shih, C., Lourenco, L., Dommelen, L.V., Krothapalli, A., "Unsteady Flow Past an Airfoil Pitching at a Constant Rate", AIAA Journal, Vol. 30, No. 5, 1992, pp. 1153-1161.

${ }^{5 .}$ Walker, J.M. and Chou, D.C., "Forced Unsteady Vortex Flows Driven by Pitching Airfoils", 19th AIAA Fluid Dynamics, Plasma and Dynamics and Lasers Conference, AIAA-87-1331.

${ }^{6 .}$ Walker, J.M., Helin, H.E., and Strickland, J.H., "An Experimental Investigation of an Airfoil Undergoing Large-Amplitude Pitching Motions", AIAA Journal, Vol.23, No.8, 1985, pp. 1141-1142.

${ }^{7}$ Walker J.M. and Chou, D.C., "Unsteady Surface Pressure Measurements on a Pitching Airfoil", AIAA Shear Flow Control Conference, AIAA-85-0532.

${ }^{8 .}$ Helin, H.E. and Walker, J.M., "Interrelated Effects of Pitch Rate and Pivot Point on Airfoil Dynamic Stall", 23rd AIAA Aerospace Sciences Meeting, AIAA-85-0130.

9. Jumper, E.J., Dimmick, R.L., and Allaire, A.J.S., "The Effect of Pitch Location on Dynamic Stall", Transactions of the ASME, Vol. 111, 1989, pp. 256-262.

10. Jumper, E.J., Schreck, S.J., and Dimmick, R.L., "Lift-Curve Characteristics for an Airfoil Pitching at Constant Rate", Journal of Aircraft, Vol. 2, No. 10, 1987, pp. 680-687.

11. Daley, D.C. and Jumper, E.J., "Experimental Investigation of Dynamic Stall for a Pitching Airfoil", Journal of Aircraft, Vol. 21, No. 10, 1984, pp. 831-832.

12. Albertson, J.A., Troutt, T.R., and Kedzie, C.R., "Unsteady Aerodynamic Forces at Low Airfoil Pitching Rates", Archive Set 340, 1988, pp. 454-462.

13. Acharya, M. and Metwally, M.H., "Unsteady Pressure Field and Vorticity Production over a Pitching Airfoil", AIAA Journal, Vol. 30, No. 2, 1992, pp. 403-411.

14. O1, M.O., "The High-Frequency, High-Amplitude Pitch Problem: Airfoils, Plates and Wings", 39th AIAA Fluid Dynamics Conference, AIAA 2009-3686.

15. Eldredge, J. D., Wang, C., and Ol, M. V.“ A Computational Study of a Canonical Pitch-up, Pitch-down Wing Maneuver," AIAA Paper 2009-3687, June 2009.

16. Grandlund, K., Ol, M.V., Garmann, D., Visbal, M. and Bernal, L.P. "Experiments and computations on abstraction of perching", AIAA Paper 2010 - 4943, June 2010.

17. Baik, Y.S., Jonathan, H.A., Bernal, L.P., Shyy, W., and Ol, M.V., "Experimental Study of a Rapidly Pitched

Flat Plate at Low Reynolds Number", 40th AIAA Fluid Dynamics Conference and Exhibit, AIAA 2010-4462.

18. Yu, H.T., Bernal, L.P, and Morrison, C., "Experimental Investigation of Pitch Ramp-Hold-Return Motion of

Flat Plates at Low Reynolds Number", AIAA Paper 2012 - 51, Jan. 2012.

19. Yu, H.T. and Bernal, L.P, "Effect of Pivot Point on Aerodynamic Force and Vortical Structure of Pitching Flat Plate Wings", AIAA Paper 2013 - 0792, Jan. 2013.

20. Visbal, M.R., "Three-Dimensional Flow Structure on a Heaving Low-Aspect-Ratio Wing", 49th AIAA Aerospace Sciences Meeting including the New Horizons Forum and Aerospace Exposition, AIAA 2011-219.

21. Yilmaz, T.O. and Rockwell, D., "Flow Structure on Finite-Span Wings due to Pitch-Up Motion", Journal of Fluid Mechanics, Vol. 691, 2012, pp518-545.

22. Anderson, J.D., Fundamentals of Aerodynamics, $5^{\text {th }}$ ed., McGraw-Hill, New York, 2011, Chaps. 4 - 5.

${ }^{23 .}$ Leishman, J.G., Principle of Helicopter Aerodynamics, $2^{\text {nd }}$ ed., Cambridge University Press, New York, 2006, Chap. 8. 